\title{
On nonlinear stability of polytropic galaxies ${ }^{1}$
}

\author{
by \\ G. WOLANSKY ${ }^{2}$ \\ Technion, 32000 Haifa, Israel
}

\begin{abstract}
We consider a certain class of stationary solutions of the Vlasov-Poisson system with a Newtonian potential. These solutions represent some spherically-symmetric, rotating galaxies obeying the generalized Emden-Fowler law. A nonlinear stability under spherically symmetric perturbations is proved. The proof is based on an adaptation of the energy-Casimir method, which implies a uniform estimate on the mass distribution of the perturbed solutions.
\end{abstract}

(C) 1999 L'Association Publications de l'Institut Henri Poincaré. Published by Elsevier B.V. All rights reserved

RÉSUMÉ. - Nous considérons une certaine classe de solutions stationnaires du système de Vlasov-Poisson avec un potentiel Newtonien. Ces solutions décrivent des galaxies tournantes, à symétrie sphérique, obéissant à la loi d'Emden-Fowler. La stabilité nonlinéaire aux perturbations sphériquement symétriques est démontrée. La preuve repose sur une adaptation de la méthode d'énergie-Casimir, laquelle implique une estimation uniforme sur la distribution de masse des solutions perturbées.

C 1999 L'Association Publications de l'Institut Henri Poincaré. Published by Elsevier B.V. All rights reserved

\footnotetext{
A.M.S. classification numbers: 70 k 20, 70 D 05.

' Supported by the fund for the promotion of the research at the Technion and the U.S-Israel BSF.

2 Tel. 972-4-294194; fax: 972-4-8324654; e.mail: mar0110@math.technion.ac.il 


\section{INTRODUCTION AND REPRESENTATION OF THE MAIN RESULT}

We study the nonlinear stability of a class of stationary, spherically symmetric solutions of the Vlasov Poisson (V-P) equation in six dimensional phase space $\left(\mathbb{R}_{\mathbf{x}}^{3}\right.$-position; $\mathbb{R}_{\mathbf{v}}^{3}$-velocity):

$$
\frac{\partial f}{\partial t}+\mathbf{v} \cdot \nabla_{\mathbf{x}} f-\nabla_{\mathbf{x}} U_{f}(\mathbf{x}, t) \cdot \nabla_{\mathbf{v}} f=0
$$

$$
\Delta_{\mathbf{x}} U_{f}(\mathbf{x}, t)=\rho_{f}(\mathbf{x}, t)
$$

where

$$
\rho_{f}(\mathbf{x}, t) \equiv \int f(\mathbf{x}, \mathbf{v}, t) d^{3} \mathbf{v},\{\mathbf{x}, \mathbf{v}\} \in \mathbb{R}^{6}, t \geq 0
$$

Denote

$$
r=|\mathbf{x}|, \quad v=|\mathbf{v}|
$$

A function $f=f(\mathbf{x}, \mathbf{v})$ is called spherically symmetric if $f=g(r, v, \mathbf{x} \cdot \mathbf{v})$. The "Jeans Theorem" implies that any stationary solution $f_{0}$ of the V-P system must take the form

$$
f_{0}=H(E, L)
$$

where

$$
E=E_{f}=v^{2} / 2+U_{f}(r)
$$

is the local energy and

$$
L=v^{2} r^{2}-(\mathbf{v} \cdot \mathbf{x})^{2}
$$

is the modulus of the angular momentum. The relation (5) was rigorously proved provided the spherical symmetry of $f_{0}$ is pre-assumed [4].

The family of stationary solutions under consideration corresponds to models of polytropic gas spheres, namely,

(8) $f_{0}=K\left(E_{0}-E\right)^{\mu} L^{k}, E_{0} \in \mathbb{R}^{-} ; \mu>-1, k>-1, \mu+k+3 / 2 \geq 0$

and $K>0$ is a normalization factor. 
With the substitution

$$
U_{0}(r)=E_{0}-\phi(c r) ; c=c(K)
$$

where $U_{0} \equiv U_{f_{0}}$, equation (2), (3), (8) reduces to the Generalized EmdenFowler Equation $(\mathrm{E}-\mathrm{F})$ on $\mathbb{R}^{+}$:

$$
\frac{1}{r^{2}} \frac{d}{d r}\left(r^{2} \frac{d}{d r} \phi(r)\right)+r^{2 k} \phi_{+}^{n}(r)=0
$$

where $(\cdot)_{+}$stands for the positive part of the argument and

$$
n \equiv 3 / 2+\mu+k \text {. }
$$

One may always choose the normalization constant $K$ so that $c(K)=1$, without limiting the generality. Under the above choice, the mass density distribution $\rho_{0} \equiv \rho_{f_{0}}$ corresponding to the stationary $f_{0}$ is given by

$$
\rho_{0}(r)=-\frac{1}{r^{2}} \frac{d}{d r}\left(r^{2} \frac{d}{d r} \phi_{0}(r)\right)=r^{2 k}\left[\phi_{0}\right]_{+}^{n}(r) .
$$

where $\phi_{0}$ is the corresponding solution of (10).

The class of stationary solutions $f=f_{0}$ considered in this paper are compactly supported in the phase space $\mathbb{R}_{\mathbf{x}}^{3} \times \mathbb{R}_{\mathbf{v}}^{3}$ and have a finite total mass

$$
\iint f_{0} d^{3} \mathbf{v} d^{3} \mathbf{x}<\infty
$$

The corresponding E-F solutions $\phi_{0}$ which we consider are called Esolutions. These are regular solutions of (10), satisfying

$$
\lim _{r \rightarrow 0} \phi_{0}(r) \equiv \phi_{0}(0)<\infty .
$$

The stationary solution $f_{0}$ itself is not necessarily regular since $k$ in (8) may take negative values. A survey of results on the E-F equations was given in [15]. A complete characterization of the solutions of the E-F equation was given in [4], [5], [13]. In section 2 we provide a short survey of relevant results regarding E-solutions of the E-F equation.

The stability question for stationary solutions of the Vlasov-Poisson equation in general, and for the E-F solutions, in particular, is a very intricate one. The first stability results for energy dependent distributions $f=f(E), d f / d E<0$ was introduced in the early 60's [1], [2], where lineraized stability was obtained for regular, non-rotating $(k=0)$ polytrpos in the range $0<\mu<7 / 2$. After this, a long list of publications concerning 
linear and non-linear stability was developed in the astronomical literature. A good reference for these results is given in [7] and [8]. However, there are very few rigorous mathematical proofs of stability (cf. [6]).

The essential difficulty behind a rigorous mathematical proof is the degenerate Hamiltonian structure of the Vlasov-Poisson Eq. Thus, an investigation of the spectral properties of the linearized equation and a proof of linear stability is not sufficient to guarantee Lyapunov stability. A non-linear approach, based on the energy-Casimir method, was applied by several authors [10], [9] but a successful rigorous stability, based on this method, was obtained only for certain cases of stationary solutions of the Vlasov-Poisson Eq. with electrostatic potential (the plasma case), c.f. [6], and [11]. An analogous proof for the gravitational Vlasov-Poisson Eq. is much more difficult, since the Casimir-energy functional is not convex in the latter case.

An attempt to provide a rigorous stability proof by an adaptation of the energy-Casimir method for non-trivial energy dependent equilibria of the gravitational V-P was introduced by Wan [14]. However, Wan could not overcame an essential difficulty of this method, namely, the differentiability of the functional for phase-space distributions which are not uniformly Lipschitz in the velocities. He thus had to assume a uniform Lipschitz condition for time dependent solutions, which is not supported by any known result.

A way out of this assumption is given in the present paper. The price paid, however, is in the weaker definition of stability which will be used.

We shall restrict ourselves to spherically symmetric perturbations, where $f=f(r, v, \mathbf{x} \cdot \mathbf{v})$. This assumption can be relaxed in the case $k=0$ (i.e. nonrotating Galaxies), while numerical evidence indicates that some rotating Galaxies $(k \neq 0)$ are unstable for non-spherically symmetric perturbations. This suggests that the present result is not too far from optimal.

\subsection{Formulation of the main result}

We will consider, throughout the paper, stationary E- solutions subject to

$$
0<\mu<3 / 2+k, \quad \mu \neq-(1 / 2+k) \quad \text { and } \quad k>-1
$$

which, via (11), is equivalent to

$$
n \neq 1, \quad 3 / 2+k<n<3+2 k, \quad k>-1
$$

Under the above conditions, any regular solution $\phi$ of the E-F equation induces a stationary solution $f_{0}$ which is compactly supported in the phase 
space $\mathbb{I R}^{6}$ and whose total mass is finite (c.f. section 2). Let us denote the mass of $f_{0}$ by $M_{0}$ :

$$
M_{0} \equiv \iint f_{0}(\mathbf{x}, \mathbf{v}) d^{3} \mathbf{v} d^{3} \mathbf{x}
$$

Before presenting our main result, we need some technical definitions:

- $C_{b}^{s}$ is the set of continuous functions $f=f(\mathrm{x}, \mathrm{v})$ on $\mathbb{R}^{6}=\mathbb{R}_{\mathrm{x}}^{3} \times \mathbb{R}_{\mathrm{v}}^{3}$ having a compact support, which are spherically symmetric, i.e: $f=g(x, v, \mathbf{x} \cdot \mathbf{v})$.

- $C_{b}^{s,+}$ is the cone of nonnegative functions in $C_{b}^{*}$. For given $M>0$, $C_{b, M}^{s,+}$ is the hyperplane of functions $f \in C_{b}^{s,+}$ which satisfy the integral constraint

$$
\iint f d^{3} \mathbf{v} d^{3} \mathbf{x}=M
$$

- Given any $\mu>0$ and a real $k$, let $C_{k}^{s}\left(\mu, k_{i}\right)$ be the space of functions $f \in C_{b}^{s}$ satisfying

$$
\begin{gathered}
\|\| f \| \equiv\left(\iint\left|v^{2} r^{2}-(\mathbf{v} \cdot \mathbf{x})^{2}\right|^{-k / \mu}|f(\mathbf{x}, \mathbf{v})|^{1+1 / \mu} d^{3} \mathbf{v} d^{3} \mathbf{x}\right)^{\frac{\mu}{1+\mu}} \\
+\frac{1}{2} \iint v^{2}|f| d^{3} \mathbf{v} d^{3} \mathbf{x}<\infty
\end{gathered}
$$

- Analogously, $C_{b}^{s,+}(\mu, k)$ and $C_{b, M}^{s,+}(\mu, k)$ are given by $C_{b}^{s}(\mu, k) \cap C_{b}^{s,+}$ and $C_{b}^{s}(\mu, k) \cap C_{b, M}^{s,+}$, respectively.

- $\mathbf{B}(\mu, k)$ is the Banach space obtained by a completion of $C_{b}^{s}(\mu, k)$ with respect to the norm (17).

- $\mathbf{B}^{+}(\mu, k)$ is the cone of a.e nonnegative functions in $\mathbf{B}(\mu, k)$, obtained by the completion of $C_{b}^{s,+}(\mu, k)$ with respect to the underlying norm. Similarly, $\mathbf{B}_{M}^{+}(\mu, k) \subset \mathbf{B}^{+}(\mu, k)$ is the completion of $C_{b, M}^{s,+}(\mu, k)$.

Remark 1.1. - Notice that $C_{b}^{s}(\mu, k)=C_{b}^{s}$ if $\mu>k$, since the kernel $\left|v^{2} r^{2}-(\mathbf{v} \cdot \mathbf{x})^{2}\right|^{\alpha}$ is locally integrable for $\alpha>-1$. The corresponding Banach space $\mathbf{B}(\mu, k)$ still depends, of course, on the particular values of the parameters $\mu$ and $k$.

Notation. - In this paper we shall fix a pair of parameters $\mu, k$ subjected to (14). We will, therefore, refer to $\mathbf{B}(\mu, k), \mathbf{B}^{+}(\mu, k)$ and $\mathbf{B}_{M}^{+}(\mu, k)$ as $\mathbf{B}, \mathbf{B}^{+}$and $\mathbf{B}_{M}^{+}$, respectively. If $M=M_{0}$ is given by (16), then we refer to $\mathbf{B}_{M_{0}}^{+}$as $\mathbf{B}_{0}^{+}$. 
By definition, the spatial distribution

$$
\rho_{f}(\mathbf{x})=\int f(\mathbf{x}, \mathbf{v}) d^{3} \mathbf{v}
$$

is well defined and nonnegative for any $f \in C_{b}^{s,+}$. Moreover, $\rho_{f}=\rho_{f}(r)$.

The Mass Function $\mathcal{M}_{f}(r)$ associated with $\rho_{f}$ measures the total mass due to $f$ contained in the ball of radius $r$ in the position space $\mathbb{R}_{\mathbf{x}}^{3}$

$$
\mathcal{M}_{f}(r)=4 \pi \int_{0}^{r} s^{2} \rho_{f}(s) d s .
$$

The main result to be proved in this paper is:

Тнеокем 1.1. - Let $f_{0}$ be an E-F stationary solution (8) subjected to (14), and induced by a regular (E-solution) of the E-F equation (12). Then $f_{0}$ is stable in the following sense: $\forall \varepsilon>0$ sufficiently small $\exists \delta>0$ so that for any initial data $\hat{f} \in \mathbf{B}_{0}^{+} \cap C^{1}\left(\mathbb{R}^{6}\right)$ which satisfies

$$
\left\|\hat{f}-f_{0}\right\| \leq \delta
$$

it follows that

$$
\sup _{t>0} \sup _{r>0}\left|\mathcal{M}_{f(\cdot, t)}(r)-\mathcal{M}_{f_{0}}(r)\right| \leq \varepsilon
$$

where $f(\cdot, t)$ is the solution of $(1-3)$ subjected to the initial data $\hat{f}$.

Remark 1.2. - The existence and uniqueness of classical solutions for $C^{1}$ initial data with bounded support in the phase space $\mathbb{R}^{6}$ was obtained by several authors (see, e.g. [12]). In the class of spherically symmetric functions, the first existence result was given in [3]. We restricted the initial data $\hat{f}$ to $C^{1}$ in order to guarantee the existence of such classical solutions.

Remark 1.3. - Theorem 1.1 implies a weaker definition of nonlinear stability since it uses the semi-metric (with respect to $\mathbf{B}$ ) defined in (21) to bound the perturbations uniformly in time, while it requires the bound (20) on the initial perturbations in terms of the stronger metric induced by the norm $|\mathrm{I} \cdot| \mathrm{II}$. Moreover, we restrict ourselves to mass conserving perturbations $\hat{f} \in \mathbf{B}_{0}^{+}$.

Remark 1.4. - If $\mu>k$ then, by Remark 1.1, condition (20) in Theorem 1.1 can be replaced by

$$
\sup _{\mathbb{R}^{6}}\left|\hat{f}-f_{0}\right|<\delta
$$


for some $\delta^{\prime}>0$ which depends on the support of $\hat{f}$. Employing the additional condition (14), we may replace (20) by (22) provided $\mu, k$ are restricted to the range

$$
n \neq 1, \quad 2 k+3>n>2 k+3 / 2, \quad k>-1
$$

equivalent to

$$
k+3 / 2>\mu>\max \{k, 0\} ; k>-1, \quad \mu \neq(-1 / 2+k) .
$$

\subsection{The energy-Casimir method}

The nonlinear stability result for $f_{0}$ (Theorem 1.1) will be obtained provided

THEOREM 1.2

(1) If $\hat{f} \in \mathbf{B}_{0}^{+} \cap C^{1}\left(\mathbb{R}^{6}\right)$ with a compact support in $\mathbb{R}^{6}$, then there exists a unique classical solution $f(\cdot, t)$ of the $V$-P system subjected to the initial data $f(\cdot, 0)=\hat{f}$ and $f(\cdot, t) \in \mathbf{B}_{0}^{+}$for any $t \geq 0$.

(2) Under the conditions of Theorem 1.1, $f_{0} \in \mathbf{B}_{0}^{+}$.

(3) There exists a functional $D \in C\left(\mathbf{B}_{0}^{+}\right)$which is invariant with respect to classical solutions of the V-P system, subjected to the initial data $\hat{f}$ in (1).

(4) Given $f \in \mathbf{B}_{0}^{+}$, then the function $M_{f}$ defined via (19) is absolutely continuous, monotone non-decreasing and satisfy $\mathcal{M}_{f}(0)=0$.

(5) There exists a positive-valued function $\delta^{\prime}=\delta^{\prime}(\varepsilon)$ for all $\varepsilon>0$ small enough, where

$$
\mathcal{D}(f)-\mathcal{D}\left(f_{0}\right)>\delta^{\prime}\left(\operatorname{Sup}_{r>0}\left|\mathcal{M}_{f}(r)-\mathcal{M}^{0}(r)\right|\right)
$$

for any $f \in \mathbf{B}_{0}^{+}$. Here $\mathcal{M}^{0} \equiv \mathcal{M}_{f_{0}}$.

Below we describe the energy-Casimir functional $\mathcal{D}$ which is used in Theorem $1.2(3,5)$.

We associate the kinetic energy with a linear functional

$$
\mathcal{E}_{k}(f)=\frac{1}{2} \iint v^{2} f(\mathbf{x}, \mathbf{v}) d^{3} \mathbf{v} d^{3} \mathbf{x}
$$

and the potential energy with the quadratic functional

$$
\mathcal{E}_{p}(f)=\frac{1}{2} \int \rho_{f} U_{f} d^{3} \mathbf{x}
$$

where $\rho_{f}$ is given in (3) and $U_{f}$ is given by (2).

Define the energy-Casimir functional:

$$
\begin{gathered}
\mathcal{D}(f)-K^{-1 / \mu} \frac{\mu}{1+\mu} \iint f^{1+1 / \mu}\left|v^{2} r^{2}-(\mathbf{v} \cdot \mathbf{x})^{2}\right|^{-k / \mu} d^{3} \mathbf{v} d^{3} \mathbf{x} \\
+\mathcal{E}_{k}(f)+\mathcal{E}_{p}(f) .
\end{gathered}
$$

Vol. $16, n^{\circ} 1-1999$ 
It is rather easy to ublain the proof of the main result (Theorem 1.1) granted Theorem 1.2 (c.f. section 5.2). The full proof of Theorem 1.2 is the subject of the most of this paper. It is, however, illuminating, at this point, to show that $\mathcal{D}$ is indeed an invariant functional for the V-P system, provided the initial data satisfies the condition of Theorem 1.2 (1).

The existence of classical solutions $f(\cdot, t)$ within the class $C^{1} \cap C_{b}^{s,+}$ with bounded supports is a known result [3]. Such classical solutions admit a finite total energy $\mathcal{E}(f)=\mathcal{E}_{k}(f)+\mathcal{E}_{p}(f)$ which is preserved along the solution $f=f(\cdot, t)$ (see, e.g [12]). Moreover, classical solutions are renormalized ones, i.e. preserve the functionals of the form

$$
\iint J(f) d^{3} \mathbf{v} d^{3} \mathbf{x}
$$

where $J$ is a real-valued function for which the integral (29) makes sense. If, in addition, the initial data $\hat{f}$ is spherically symmetric, then the solution $f(\cdot, t)$ is spherically symmetric as well $\forall t>0$ and the sample trajectories of the particles are subjected to a (perhaps nonstationary) central force field. Thus the angular momentum $L$ is conserved along the trajectories. This implies that (29) is generalized into

$$
\iint J(f, L) d^{3} \mathbf{v} d^{3} \mathbf{x}
$$

where $J(\cdot, \cdot)$ is, again, any function for which the integral (30) makes sense.

The motivation for the particular choice of $J$ in functional $\mathcal{D}$ is as follows: Let

$$
\Psi(\lambda, L) \equiv \frac{K}{\mu+1} \lambda_{+}^{\mu+1} L^{k}
$$

The function $\Psi$ is the primitive (with respect to $E_{0}-E$ ) of the E-F distribution law (8). Since $\mu>0$ by assumption (14), then $\Psi$ is convex with respect to the first variable. The function

$$
J(\zeta, L) \equiv K^{-1 / \mu} \frac{\mu}{1+\mu} \zeta^{1+1 / \mu} L^{-k / \mu} \text { if } \zeta \geq 0 ; J(\zeta, L)=\infty \text { if } \zeta<0
$$

is the convex-conjugate of $\Psi$ with respect to $\zeta$. Then $\mathcal{D}$ is given by

$$
\mathcal{D}(f)=\iint J(f, L) d^{3} \mathbf{v} d^{3} \mathbf{x}+\mathcal{E}(f)
$$

where $\mathcal{E}(f) \equiv \mathcal{E}_{k}(f)+\mathcal{E}_{p}(f)$. 
By equating the formal differentiation of $\mathcal{D}$ to zero subjected to the constraint hyperplane of a prescribed mass

$$
\iint f d^{3} \mathbf{v} d^{3} \mathbf{x}=M_{0}
$$

we, indeed, recover the E-F relation (8), where $E_{0}$ plays the rule of Lagrange multiplier. This indicates that the choice of $J$ as given by (32) is a necessary condition for $f_{0}$ to be a minimizer of $\mathcal{D}$ over $\mathbf{B}_{0}^{+}$.

\subsection{Organization of the paper}

The essential difficulty is the proof that the particular choice (32) for $J$ is also sufficient for part (5) of Theorem 1.2 to hold.

In section 2 we introduce some results about regular solutions of the E-F equation which are required for the proof of the main result. In section 3 we present one of the key ideas: A reduction of the energy-Casimir functional by eliminating the velocity dependent part and projecting it onto the space of mass perturbations $\rho=\rho(\mathbf{x})$ rather then $f=f(\mathbf{x}, \mathbf{v})$. The reduced functional is used in section 4 and yields a variational formulation of the E-F equation. In this section we also lay the foundation for the proof of Theorem 1.2 (5) for perturbations supported on a fixed bounded domain. In section 5 we remove this restriction and obtain uniform estimates, independent of the support of the perturbations. We then summarize all these results in section 5.2 to obtain the proof of Theorem 1.2 and, through which, the proof of the main result Theorem 1.1 .

\section{THE EMDEN-FOWLER EQUATION: AUXILIARY RESULTS}

The solutions $\phi=\phi(r)$ considered in this paper are E-solutions of the Emden-Fowler equation (10), i.e. satisfy

$$
\lim _{r \rightarrow 0} \phi(r)=\alpha \text { where } 0<\alpha<\infty
$$

A solution $\phi$ is said to be of finite mass if

$$
\lim _{r \rightarrow \infty} r^{2} \phi^{\prime}(r)>-\infty
$$

and it is of finite radius if

$$
\exists R>0 \text { for which } \phi(R)=0 ; \phi(r)<0 \text { for all } r>R
$$


Notice that if both finite mass (35) and finite radius (36) conditions are satisfied then

$$
r^{2} \phi^{\prime}(r)=R^{2} \phi^{\prime}(R) \text { for all } r>R
$$

The results of Lemma 2.1 and Lemma 2.2 below are distributed in [4], [3], [5] and [13]. We introduce the detailed proofs for completeness. Lemma 2.1 determines the existence of E-solutions of finite mass and radius assumed in Theorem 1.1. Lemma 2.2 characterizes the E-solution in the range (23).

The results of Lemma 2.3 and Lemma 2.4, on the other hand, could not be found explicitly in the above cited references. Lemma 2.3 is a key result in the proof of the inequality (25) for uniformly supported $f \in \mathbf{B}_{0}^{+}$. Lemma 2.4 will enable us to remove this restriction.

LEMMA 2.1. - If $n>0$ and $k>-1$ then there exists an E-solution of the $E$ - $F$ equation. If $\phi_{0}$ is an E-solution and $n \neq 1$, then all E-solutions are given by

$$
\phi_{\beta}(r)=\beta^{\frac{2 k+2}{n-1}} \phi_{0}(\beta r) ; \beta>0
$$

If, moreover, $n<2 k+3$ then all solutions of the $E-F$ equation are of finite mass and radius, namely, for any solution $\phi$ (in particular, for an E-solution) there exists $\bar{R}_{\phi}<\infty$ and $M>0$ so that $\phi(r)<0$ and $r^{2} \phi^{\prime}(r)=-M>-\infty \forall r>\bar{R}_{\phi}$.

Proof of Lemma 2.1. Following [5] and 13] we consider the transformation

$$
u(t)=-\frac{r^{2 k+2} \phi^{m}(r)}{\phi^{\prime}(r)}, v(t)=-\frac{r \phi^{\prime}(r)}{\phi(r)}, t=\ln r
$$

which reduce the E-F equation into

$$
\begin{gathered}
u^{\prime}(t)=u(t)[2 k+3-u(t)-n v(t)] \\
v^{\prime}(t)=v(t)[-1+u(t)+v(t)]
\end{gathered}
$$

in the positive quadrant $u \geq 0, v \geq 0$. The correspondence between the E-F equation and $(39,40)$ holds in the range where $\phi(r) \geq 0$.

By Proposition 3.1 in [5], an E-solution $\phi$ of the E-F equation corresponds to an orbit of $(39,40)$ which satisfy $\lim _{t \rightarrow-\infty}\{u(t), v(t)\}=P_{3}$ where $P_{3}=(2 k+3,0)$ is a critical point of $(39,40)$. The critical point $P_{3}$ is a saddle of $(39,40)$. Thus, there is a unique orbit like this. A class of 
solutions $\phi_{\beta}$ given by (37) correspond to an orbit $\{u(t), v(t)\}$ of $(39,40)$ via the transformation $t \rightarrow t+\ln \beta$. In particular, all E-solutions congruent to the single orbit of $(39,40)$ which is the unstable manifold of $P_{3}$ in the positive quadrant.

If $n<3+2 k$ and $u\left(t_{0}\right)>0, v\left(t_{0}\right)>0$ for some $t_{0} \in \mathbb{R}$, then there exists $t_{m}<\infty$ for which

$$
\lim _{t \rightarrow t_{m}} u(t)=0 ; \lim _{t \rightarrow t_{m}} v(t)=\infty
$$

This implies that any solution $\phi$ of the E-F equation must have a finite radius. It is easy to see from the E-F equation itself (taking into account $\mu>0$ ) that any solution of finite radius must also have a finite mass.

LEMMA 2.2. - If $n<3+2 k$ and $\phi$ is a solution of the E-F equation on an interval $(0, R)$ which satisfy

$$
\lim _{r \rightarrow 0} r^{2} \frac{d \phi}{d r}=0
$$

then $\phi$ can be extended on $\mathbb{R}^{+}$as an E-solution. In particular, (4I) implies (34).

Proof of Lemma 2.2. - Any solution $\phi$ of the E-F on an interval can be extended to an entire solution on $\mathbb{R}^{+}$. This is immediately obtained from the proof of Lemma 2.1. To show that (41) yields (34), we first observe that (34) must hold where $\alpha>0$ (possibly $\alpha=\infty$ ). The later possibility is excluded in the range $n<3+2 k$ by Proposition 3.3 in [5] ${ }^{3}$.

LEMMA 2.3. - Let $3 / 2+k<n<3+2 k$ and $n \neq 1$. Consider the boundary value problem of the $E-F$ equation on an interval $(0, R]$ corresponding to:

$$
\text { (a) } \lim _{r \rightarrow 0} r^{2} \phi^{\prime}(r)=0 ; \text { (b) } R^{2} \phi^{\prime}(R)=-\frac{M}{4 \pi}
$$

where $M>0$ is a given constant. Then there exists at most one solution for any given $M>0$.

Lemma 2.4. - Let $3 / 2+k<n<3+2 k$ and $n \neq 1$. Consider the initial value problem of the $E-F$ equation corresponding to:

$$
\phi_{\alpha, \beta}\left(R_{1}\right)=\alpha>0 ; R_{1}^{2} \phi_{\alpha, \beta}^{\prime}\left(R_{1}\right)=-\beta<0 ; R_{1}>0
$$

\footnotetext{
${ }^{3}$ Notice that our notation for $n$ corresponds to $n+m$ in [5] and $m$ corresponds to our $k$.
} Vol. $16, n^{\circ} 1-1999$. 
Assume a solution of $(10,43)$ exists on an interval $\left[R_{1}, R_{*}\right]$ for any $\alpha$, $\beta>0$. Then

$$
\lim _{\Omega \rightarrow \infty} R_{*}^{2} \phi_{\Omega, \beta}^{\prime}\left(R_{*}\right)=-\infty
$$

Proof of Lemma 2.3. - The initial condition (42-a) implies that $\phi$ is an E-solution by Lemma 2.2. All other E-solutions are given, via Lemma 2.1, by

$$
\phi_{\beta}(r) \equiv \beta^{\gamma+1} \phi(\beta r)
$$

for some $\beta>0$ where $\gamma=(2 k+2) /(n-1)$. The relation between $\beta, M$ and $R$ is given, via (42-b) by

$$
M \equiv M_{\beta}^{(R)}=-4 \pi R^{2} \beta^{\gamma+2} \phi^{\prime}(\beta R) .
$$

To prove the Lemma we show that $M_{\beta}^{(R)}$ is strictly monotone in $\beta$ for any given $R>0$. Differentiation of (46) with respect to $\beta$ yields:

$$
\frac{d}{d \beta} M_{\beta}^{(R)}=-4 \pi R^{3} \beta^{\gamma+2}\left[\frac{\gamma+2}{s} \frac{d}{d s}+\frac{d^{2}}{d s^{2}}\right] \phi(s),
$$

evalualed at $s=\beta R$.

Case $n>1$ : In this case, $\gamma>0$. Since $\phi^{\prime}(s)<0$ for any $s>0$ we obtain, by the E-F Equation (10)

$$
\frac{d}{d \beta} M_{\beta}^{(R)}>-4 \pi R^{3} \beta^{\gamma+2}\left[\frac{2}{s} \frac{d}{d s}+\frac{d^{2}}{d s^{2}}\right] \phi(s)=4 \pi s^{2 k} R^{3} \beta^{\gamma+2} \phi_{+}^{n}(s) \geq 0 .
$$

Case $n<1$ : In this case, $\gamma<0$. Substituting the E-F equation in (47) we obtain

$$
\begin{aligned}
-\frac{d}{d \beta} M_{\beta}^{(R)} & =4 \pi R^{2} \beta^{\gamma+1}\left[\gamma \frac{d}{d s} \phi-s^{2 k+1}[\phi(s)]_{+}^{n}\right] \\
& =4 \pi R^{2} \beta^{\gamma+1} \phi^{\prime}(s)[\gamma+u(s)] .
\end{aligned}
$$

where $u$ as defined in (38). Using lemma 5.2(b) in [5] we conclude that $u(s)<2 k+3$ for any $s>0$, unless $\phi$ is an 'F-Solution' (which is not our case). Hence

$$
\begin{aligned}
-\frac{d}{d \beta} M_{\beta}^{(R)} & \geq 4 \pi R^{2} \beta^{\gamma+1}\left[(2 k+3+\gamma) \frac{d}{d s} \phi(s)\right] \\
& =4 \pi R^{2} \beta^{\gamma+1} \frac{n(2 k+2)}{n-1} \frac{d}{d s} \phi(s)>0
\end{aligned}
$$

since $\phi^{\prime}(s)<0$ for $s>0$. 
Proof of Lemma 2.4. - Let us transform the solution $\phi=\phi_{\alpha, \beta}$ into

$$
\psi(r)=\phi\left(\frac{1}{r}\right)
$$

in terms of which, the E-F Equation takes the form

$$
\frac{d^{2}}{d r^{2}} \psi+r^{-(2 k+4)} \psi_{+}^{n}-0
$$

The corresponding initial value problem for $\phi$ is transformed into

$$
\psi^{\prime}\left(R_{1}^{-1}\right)=-R^{2} \phi^{\prime}\left(R_{1}\right) \equiv \beta>0 ; \quad \psi\left(R_{1}^{-1}\right)=\alpha>0
$$

Since we assumed the existence of the solution $\phi_{\alpha, \beta}$ on $\left[R_{1}, R_{*}\right]$, we may assume that the solution of (50) exists as well on the interval $\left[R_{*}^{-1}, R_{1}^{-1}\right]$. If $\phi\left(R_{*}\right)>0$ set $\vec{R}_{*}=R_{*}$. Otherwise, set $\bar{R}_{*}$ to be the root of $\phi=0$ in the interval $\left(R_{1}, R_{*}\right)$. Then

$$
-R_{*}^{2} \phi^{\prime}\left(R_{*}\right)=\psi^{\prime}\left(R_{*}^{-1}\right)=-\bar{R}_{*}^{2} \phi^{\prime}\left(\bar{R}_{*}\right)=\psi^{\prime}\left(\bar{R}_{*}^{-1}\right) \equiv s .
$$

By definition, $\psi>0$ on $\left(\bar{R}_{*}^{-1}, R_{1}^{-1}\right)$, hence $\psi^{\prime \prime}<0$ on the same interval by $(50)$. We take advantage of this and use

$$
p(r) \equiv \frac{d \psi}{d r}(r)
$$

as an independent variable. Set $r(p)$ to be the inverse function of $p(r)$, and

$$
\psi^{*}(p) \equiv \psi(r(p))
$$

Now:

$$
p(r)=\left(\frac{d}{d p} \psi^{*}\right) \frac{d p}{d r}=\left(\frac{d}{d p} \psi^{*}\right) \frac{d^{2}}{d r^{2}} \psi(r)
$$

hence the initial value problem $(50,51)$ is equivalent to:

$$
\frac{d}{d p} \psi^{*}+\operatorname{pr}_{(p)}^{2 k+4}\left[\psi^{*}\right]_{+}^{-n}=0 ; \psi^{*}(\beta)=\alpha
$$

An integration of (53) on an interval $[\beta, s]$ yields:

$$
\left[\psi^{*}(s)\right]^{n+1}=-(n+1)\left[\int_{\beta}^{s} p r_{(p)}^{2 k+4} d p\right]+\alpha^{n+1} .
$$


Since $2 k+4>0$ and $0 \leq r(p) \leq R_{1}^{-1}$ on the interval $[\beta, s]$ we estimate the integral in (54) by

$$
\int_{\beta}^{s} \operatorname{pr}_{(p)}^{2 k+4} d p \leq s(s-\beta) R_{1}^{-(2 k+4)}
$$

In particular we obtain by $(54,55)$ that

$$
\phi^{n+1}(r) \geq \alpha^{n+1}-s(s-\beta)(n+1) R_{1}^{-(2 k+4)}
$$

on the interval $\left[R_{1}, \bar{R}_{*}\right]$.

Let us suppose, in contradiction to the statement of the Lemma, that there exists a sequence $\alpha_{j} \rightarrow \infty$ along which

$$
R_{*}^{2} \phi_{\alpha_{j}, \beta}^{\prime}\left(R_{*}\right)=\bar{R}_{*}^{2} \phi_{\alpha_{j}, \beta}^{\prime}\left(\bar{R}_{*}\right) \equiv-s_{j}<0 ; \sup _{j \geq 1} s_{j}<\infty
$$

Then (56) implies that $\phi_{\alpha_{j}, \beta}>0$ on $\left[R_{1}, \bar{R}_{*}\right]$ if $\alpha_{j}$ is sufficiently large. This, in turn, implies that $\bar{R}_{*}=R_{*}$ by definition. Moreover, we obtain by the E-F equation

$$
R_{*}^{2} \phi_{\alpha_{j}, \beta}^{\prime}\left(R_{*}\right)=R_{1}^{2} \phi_{\alpha_{j}, \beta}^{\prime}\left(R_{1}\right)-\int_{R_{1}}^{R_{*}} r^{2(k+1)}\left[\phi_{\alpha_{j}, \beta}\right]_{+}^{n} d r
$$

$\leq-\beta-\left[\alpha_{j}^{n+1}-s_{j}\left(s_{j}-\beta\right)(n+1) R_{1}^{-(2 k+4)}\right]^{\frac{n}{n+1}} \int_{R_{1}}^{R_{*}} r^{2(k+1)} d r \rightarrow-\infty$

since $\alpha_{j} \rightarrow \infty$ while $s_{j}$ are uniformly bounded by assumption. Then, we obtain a contradiction.

\section{REDUCTION OF THE ENERGY-CASIMIR FUNCTIONAL}

Let us define

$\mathbf{X} \equiv\{\mathcal{M}:[0, \infty) \rightarrow R$, absolutely continuous on $[0, \mathrm{R}] \forall R>0, \mathcal{M}(0)=0\}$

equipped with the norm

$$
\|\mathcal{M}\| \equiv\left\{\int_{0}^{\infty} r \frac{-2(k+1)}{n}\left|\frac{d \mathcal{M}}{d r}\right|^{\frac{n+1}{n}} d r\right\}^{\frac{n}{n+1}}
$$

Lemma 3.1 below is a corner stone in the reduction of the energy-Casimir functional, to be used in the rest of the paper. 
LEMMA 3.1. -. The mapping

$$
f \in \mathbf{B} \longrightarrow \mathcal{M}_{f} \in \mathbf{X}
$$

(c.f. notation below remark 1.1) is well defined and bounded under the underlying norms. Moreover

$$
\iint J(f, L) d^{3} \mathbf{v} d^{3} \mathbf{x}+\frac{1}{2} \iint v^{2} f d^{3} \mathbf{v} d^{3} \mathbf{x} \geq \frac{1}{(4 \pi)^{1 / n}} \cdot \frac{n}{n+1}\left\|\mathcal{M}_{f}\right\|^{\frac{n+1}{n}}
$$

$\forall f \in \mathbf{B}$, where $J$ is given by (32).

Proof of Lemma 3.1. - Since B was defined as a completion of the space $C_{b}^{s}(\mu, k)$, it is enough to prove the Lemma for $f \in C_{b}^{s}(\mu, k)$. Let $\rho_{f}$ be defined as in (18). Set

$$
\mathcal{P}_{\rho_{f}} \equiv\left\{h \in C_{b}^{s}(\mu, k) ; \int h(\mathbf{x}, \mathbf{v}) d^{3} \mathbf{v}=\rho_{f}(\mathbf{x}) \forall \mathbf{x} \in R^{3}\right\}
$$

Define

$$
\bar{J}\left(\rho_{f}\right)=\inf _{h \in \mathcal{P}_{\rho_{f}}}\left\{\iint J(h, L) d^{3} \mathbf{v} d^{3} \mathbf{x}+\frac{1}{2} \iint v^{2} h d^{3} \mathbf{v} d^{3} \mathbf{x}\right\}
$$

We shall prove that

$$
\bar{J}(\rho) \geq \frac{4 \pi n}{n+1} \int_{0}^{\infty} \rho^{(n+1) / n} r^{-2(k-n) / n} d r
$$

In particular, the right-hand-side of (64) is finite if $\rho=\rho_{f}(r)$ for some $f \in C_{b}^{s}(\mu, k)$. A direct computation, using $\mathcal{M}_{f}^{\prime}(r)=4 \pi r^{2} \rho_{f}(r)$ (c.f. (19)) yields that the r.h.s of (64) is identical to the r.h.s of $(61)$.

Proof of (64). - Define the real-valued function $\Phi$ on $\mathbb{R}^{+} \times \mathbb{R}^{3}$ :

$$
\Phi(s, \mathbf{x}) \equiv \inf _{\zeta \in P_{s}}\left\{\int J(\zeta, L) d^{3} \mathbf{v}+\frac{1}{2} \int v^{2} \zeta d^{3} \mathbf{v}\right\}
$$

where $P_{s}$ stands for the functional space:

(66)

$$
P_{s} \equiv\left\{\zeta=\zeta(\mathbf{v}) ; \zeta \geq 0 \text { a.s is measurable on } \mathbb{R}^{3} \text { and } \int \zeta d^{3} \mathbf{v}=s\right\}
$$

Define also

$$
\Phi(s, \mathbf{x}) \equiv \infty \text { if } s<0
$$

Vol. 16, n ${ }^{\circ}$ 1-1999. 
which is consistent with (32). By (63)

$$
\bar{J}(\rho) \geq \int \Phi(\rho(\mathbf{x}), \mathbf{x}) d^{3} \mathbf{x} .
$$

The function $\Phi(s, \mathbf{x})$ is evidently convex with respect to the first variable $s$, for any fixed $\mathrm{x} \in R_{\mathrm{x}}^{3}$. Let us compute the convex conjugate $\bar{\Psi}$ of $\Phi$ :

$$
\begin{aligned}
\bar{\Psi}(\lambda, \mathbf{x}) \equiv \sup _{s \in R}[\lambda s-\Phi(s, \mathbf{x})] \\
=\sup _{s \in \mathbb{R}^{+}}\left[\sup _{\zeta \in P_{s}}\left[\int\left(\lambda-\frac{1}{2} v^{2}\right) \zeta d^{3} \mathbf{v}-\int J(\zeta, L) d^{3} \mathbf{v}\right]\right] \\
=\sup _{\zeta \in Q_{*}}\left[\int\left(\lambda-\frac{1}{2} v^{2}\right) \zeta d^{3} \mathbf{v}-\int J(\zeta, L) d^{3} \mathbf{v}\right]
\end{aligned}
$$

where

$$
Q_{*} \equiv \cup_{s \in \mathbb{R}^{+}} P_{s}=\left\{\zeta=\zeta(\mathbf{v}) \geq 0 \text { a.s integrable over } \mathbb{R}^{3}\right\}
$$

The right hand side of (69) is evaluated at $\zeta_{\lambda, \mathbf{x}}=\zeta_{\lambda, \mathbf{x}}(\mathbf{v})$ which satisfies $J_{\zeta}\left(\zeta_{\lambda, \mathbf{x}}, L\right)=\lambda-1 / 2 v^{2}$, namely

$$
\zeta_{\lambda \cdot \mathbf{x}}(\mathbf{v})=K L^{k}\left(\lambda-\frac{1}{2} v^{2}\right)_{+}^{\mu}
$$

It is easy to see that $\zeta_{\lambda, \mathbf{x}} \in Q_{*}$. In fact, $L^{k}$ is locally integrable as a function of $\mathbf{v}$ for any $\mathbf{x} \in \mathbb{R}^{3}$ and $\zeta_{\lambda . \mathbf{x}}$ is supported in the ball $v \leq \sqrt{2 \lambda}$. Thus, the r.h.s of $(69)$ is written as

$$
\int \sup _{q \geq 0}\left[\left(\lambda-\frac{1}{2} v^{2}\right) q-J(q, L)\right] d^{3} \mathbf{v} .
$$

The integrand in (72) is nothing but the convex conjugate of $J(q, L)$ with respect to $q$, evaluated at $\lambda-1 / 2 v^{2}$. It is given by $\Psi\left(\lambda-1 / 2 v^{2}, L\right)$ (c.f. (31)). By a direct calculation we obtain

$$
\bar{\Psi}(\lambda, \mathbf{x})=\int \Psi\left(\lambda-\frac{1}{2} v^{2}, L\right) d^{3} \mathbf{v}=\frac{1}{n+1} r^{2 k} \lambda_{+}^{n+1}
$$

where the normalization constant $K$ is eliminated by the integration ${ }^{4}$.

\footnotetext{
${ }^{4}$ In fact, it was design exactly for this purpose.
} 
The involution property of the convex conjugacy relation leads to the explicit form of $\Phi(s, \mathbf{x})$

$$
\Phi(s, \mathbf{x})=\sup _{\lambda \in R}[\lambda s-\bar{\Psi}(\lambda, r)]=\frac{n}{n+1} r^{-2 k / n} s^{(n+1) / n}
$$

and (64) follows from (68) and (73).

Lemma 3.2. $-f_{0} \in \mathbf{B}_{0}^{+}$and the inequality (61) turns into an equality for $f=f_{0}$.

Proof of Lemma 3.2. - The first statement, namely $f_{0} \in \mathbf{B}_{0}^{+}$, follows by a direct substitution of $f_{0}=K\left(E_{0}-E\right)_{+}^{\mu} L^{k}$ in the norm (17). The first term turns out to be

$$
K\left(\iint L^{k}\left(E-E_{0}\right)_{+}^{\mu+1} d^{3} \mathbf{v} d^{3} \mathbf{x}\right)^{\mu /(1+\mu)}
$$

which is finite since $L^{k}$ is locally integrable for $k>-1$ and $\left(E-E_{0}\right)_{+}$ is compactly supported in $\mathbb{R}^{6}$. The second (kinetic energy) term is finite for the same reason.

We now prove the equality in (61). Given $\mathbf{x} \in \mathbb{R}_{\mathbf{x}}^{3}$, set $\zeta_{\mathbf{x}}(\mathbf{v}) \equiv$ $f_{0}(\mathbf{x}, \mathbf{v})^{5}$. Then $\zeta_{\mathbf{x}} \in P_{\rho_{0}(\mathbf{x})}$ where $P_{s}$ as defined in (66). It is enough to show

$$
\Phi\left(\rho_{0}(\mathbf{x}), \mathbf{x}\right)=\int\left(J\left(\zeta_{\mathbf{x}}, L\right)+\frac{1}{2} v^{2} \zeta_{\mathbf{x}}\right) d^{3} \mathbf{v} .
$$

Indeed, by (63)

$$
\begin{gathered}
\bar{J}\left(\rho_{0}\right)=\inf _{h \in \mathcal{P}_{f_{0}}} \iint\left(J(h, L)+\frac{1}{2} v^{2} h\right) d^{3} \mathbf{v} d^{3} \mathbf{x} \\
\geq \int_{\mathbb{R}_{\mathbf{x}}^{3}}\left[\int \inf _{\zeta \in P_{P_{0}(\mathbf{x})}} \int\left(J(\zeta, L)+\frac{1}{2} v^{2} \zeta\right) d^{3} \mathbf{v}\right] d^{3} \mathbf{x}=\int \Phi\left(\rho_{0}, \mathbf{x}\right) d^{3} \mathbf{x} \\
\text { by }(74)=\iint\left(J\left(f_{0}, L\right)+\frac{1}{2} v^{2} f_{0}\right) d^{3} \mathbf{v} d^{3} \mathbf{x} \geq \bar{J}\left(\rho_{0}\right)
\end{gathered}
$$

This implies that the last inequality in (75) is an equality, and the Lemma follows.

Proof of $(74)$. - Since $J(\cdot, L)$ is convex with respect to the first variable, we have:

$\forall \zeta \in P_{\rho_{0}(\mathbf{x})}: J(\zeta, L)+\frac{1}{2} v^{2} \zeta \geq J\left(\zeta_{\mathbf{x}}, L\right)+\frac{1}{2} v^{2} \zeta_{\mathbf{x}}+\frac{\partial}{\partial \zeta} J\left(\zeta_{\mathbf{x}}, L\right) \omega+\frac{1}{2} v^{2} \omega$

\footnotetext{
${ }^{5}$ Notice that $\zeta_{\mathbf{x}}=\zeta_{\lambda, \mathbf{x}}$ defined in (71) provided $\lambda=-U_{0}(\mathbf{x})$.
}

Vol. $16, n^{\circ} 1-1999$ 
where $\omega=\zeta-\zeta_{\mathrm{x}}$. Hence

$$
\begin{gathered}
\int\left(J(\zeta, L)+\frac{1}{2} v^{2} \zeta\right) \geq \int\left(J\left(\zeta_{\mathbf{x}}, L\right)+\frac{1}{2} v^{2} \zeta_{\mathbf{x}}\right) d^{3} \mathbf{v} \\
+\int\left[K^{-\frac{1}{\mu}}\left[\zeta_{\mathbf{x}}\right]_{+}^{\frac{1}{\mu}} L^{-\frac{k}{\mu}}+\frac{1}{2} v^{2}\right] \omega d^{3} \mathbf{v}
\end{gathered}
$$

We now show that the second integral on the r.h.s of (76) is nonnegative. Set

$$
\left.\begin{array}{l}
S_{1}=\left\{\mathbf{v} \in \mathbb{R}_{\mathbf{v}}^{3} ; E_{0}-\frac{1}{2} v^{2}-U_{0}(\mathbf{x}) \geq 0\right. \\
S_{2}=\left\{\mathbf{v} \in \mathbb{R}_{\mathbf{v}}^{3} ; E_{0} \quad \frac{1}{2} v^{2} \quad U_{0}(\mathbf{x})<0\right.
\end{array}\right\}
$$

Since $\zeta_{\mathbf{x}}=K\left[E_{0}-1 / 2 v^{2}-U_{0}(\mathbf{x})\right]_{+}^{\mu} L^{k}$, the term in square brackets in (76) is written as

$$
[\ldots]=\left[E_{0}--U_{0}(\mathbf{x})\right]_{+}+\frac{1}{2} v^{2}= \begin{cases}E_{0}-U_{0}(\mathbf{x}) & \text { if } \mathbf{v} \in S_{1} \\ \frac{1}{2} v^{2} & \text { if } \mathbf{v} \in S_{2}\end{cases}
$$

Hence

$$
\begin{aligned}
& \int\left[K^{-\frac{1}{\mu}}\left[\zeta_{\mathbf{x}}\right]_{+}^{\frac{1}{\mu}} L^{-\frac{k}{\mu}}+\frac{1}{2} v^{2}\right] \omega d^{3} \mathbf{v} \\
& =\left(E_{0}-U_{0}(\mathbf{x})\right) \int_{S_{1}} \omega(\mathbf{v}) d^{3} \mathbf{v}+\frac{1}{2} \int_{S_{2}} v^{2} \omega(\mathbf{v}) d^{3} \mathbf{v} \\
& =\int_{S_{2}}\left(\frac{1}{2} v^{2}+U_{0}(\mathbf{x})-E_{0}\right) \omega(\mathbf{v}) d^{3} \mathbf{v}
\end{aligned}
$$

where the last equality in (77) follows from

$$
\int_{\mathbb{R}^{6}} \omega(\mathbf{v}) d^{3} \mathbf{v}=0 \Longrightarrow \int_{S_{1}} \omega(\mathbf{v}) d^{3} \mathbf{v}=-\int_{S_{2}} \omega(\mathbf{v}) d^{3} \mathbf{v}
$$

Now, $\omega \geq 0$ on $S_{2}$ since $0 \leq \zeta(\mathbf{v})=\zeta_{\mathbf{x}}(\mathbf{v})+\omega(\mathbf{v})$ and $\zeta_{\mathbf{x}}(\mathbf{v})=0$ for $\mathbf{v} \in S_{2}$ by definition. In addition, $1 / 2 v^{2}+U_{0}(\mathbf{x})-E_{0}>0$ on $S_{2}$ by definition. This implies that the r.h.s of (77) is nonnegative. In particular, $\zeta_{\mathbf{x}} \in P_{\rho_{0}(\mathbf{x})}$ is a minimizer of (65) at $s=\rho_{0}(\mathbf{x})$, and (74) follows.

Let $\Gamma^{M}$ be the image of $\mathbf{B}_{M}^{+}$under the mapping (60). That is:

$$
\mathcal{M} \in \Gamma^{M} \text { iff } \mathcal{M}=\mathcal{M}_{f} \text { for some } f \in B_{M}^{+}
$$

By definition, $\Gamma^{M}$ consists of monotone non-decreasing functions which satisfy

$$
\lim _{r \rightarrow \infty} \mathcal{M}(r)=M ; \mathcal{M}(0)=0
$$


and $\|\mathcal{M}\|<\infty$ by Lemma 3.1. We denote $\Gamma^{M_{0}} \equiv \Gamma^{0}$.

Lemma 3.3. - If $\mathcal{M} \in \Gamma^{M}$ for some $M>0$, then

$$
\mathcal{M}(r) \leq\left(\frac{\|\mathcal{M}\|}{2 k+3}\right) r^{\frac{2 k+3}{n+1}}
$$

for all $r \geq 0$.

Notice that $k>-1$ and $n>1 / 2$ by (15), hence the exponent $(2 k+3) /(n+1)$ in (79) is positive.

Proof of Lemma 3.3. - A simple application of the Holder inequality yields:

$$
\begin{gathered}
\mathcal{M}(r)=\int_{0}^{r} \frac{d}{d t} \mathcal{M}(t) d t \\
=\int_{0}^{r} t^{-2(k+1) /(n+1)} \cdot t^{2(k+1) /(n+1)} \cdot\left(\frac{d}{d t} \mathcal{M}\right) d t \\
\leq\left[\int_{0}^{r}\left|\frac{d}{d t} \mathcal{M}(t)\right|^{(n+1) / n} \cdot t^{-2(k+1) / n} d t\right]^{n /(n+1)} \cdot\left[\int_{0}^{r} t^{2(k+1)} d t\right]^{1 /(n+1)} \\
=\left(\frac{\|\mathcal{M}\|}{2 k+3}\right) \cdot r^{(2 k+3) /(n+1)}
\end{gathered}
$$

We now turn our attention to the potential energy $\mathcal{E}_{p}$ defined in (27).

LEMMA 3.4. - Given $f \in C_{b, M}^{s,+}$, the potential energy $\mathcal{E}_{p}(f)$ is written, in terms of $\mathcal{M}=\mathcal{M}_{f}$, by

$$
\mathcal{E}(\mathcal{M}) \equiv \mathcal{E}_{p}(f)=-\frac{1}{8 \pi} \int_{0}^{\infty} \frac{\mathcal{M}^{2}(r)}{r^{2}} d r
$$

Proof of Lemma 3.4. - By definition

$$
\mathcal{E}_{p}(f)=\frac{1}{2} \int_{\mathbb{R}^{3}} \rho_{f}(\mathbf{x}) U_{f}(\mathbf{x}) d^{3} \mathbf{x}=2 \pi \int_{0}^{\infty} r^{2} \rho(r) U_{\rho}(r) d r .
$$

Apply (2) and use integration by parts to obtain

$$
\mathcal{E}_{p}(f)=2 \pi \int_{0}^{\infty} \frac{d}{d r}\left(r^{2} \frac{d U_{\rho}}{d r}\right) U_{\rho} d r=-2 \pi \int_{0}^{\infty} r^{2}\left[\frac{d U_{\rho}}{d r}\right]^{2} d r
$$

where, for the validity of the integration by parts, we used the regularity of $\rho=\rho_{f}$ (where $f \in C_{b, M}^{s,+}$ ), and the asymptotics of the corresponding Newtonian potential

$$
\lim _{r \rightarrow \infty} U_{\rho}(r)=0 ; \lim _{r \rightarrow \infty} r^{2} \frac{d U_{\rho}}{d r}=-\frac{M}{4 \pi} .
$$

Vol. 16, $n^{\circ}$ 1-1999. 
The Lemma follows by using $\mathcal{M}_{\rho}(r)=-4 \pi r^{2} U_{\rho}^{\prime}(r)$.

LEMma 3.5. $-\mathcal{E}$ can be extended as a continuous functional over $\Gamma^{M}$ for any $M>0$.

Proof of Lemma 3.5. - Let $\mathcal{M}_{i} \in \Gamma^{M}, i=1,2$. Then, for any $R>0$

$$
\begin{aligned}
& \left|\mathcal{E}\left(\mathcal{M}_{1}\right)-\mathcal{E}\left(\mathcal{M}_{2}\right)\right| \leq \frac{1}{8 \pi} \int_{0}^{R} \frac{\left(\mathcal{M}_{1}-\mathcal{M}_{2}\right)^{2}}{r^{2}} d r \\
& \quad+\frac{1}{8 \pi} \int_{R}^{\infty} \frac{\left(\mathcal{M}_{1}-\mathcal{M}_{2}\right)^{2}}{r^{2}} d r \\
& \leq \frac{\left\|\mathcal{M}_{1}-\mathcal{M}_{2}\right\|}{8 \pi(2 k+3)} \int_{0}^{R} r^{\frac{2(2 k+3)}{n+1}-2} d r+\frac{M^{2}}{8 \pi R} \\
& =\frac{n+1}{4 k+5-n} \frac{\left\|\mathcal{M}_{1}-\mathcal{M}_{2}\right\|}{8 \pi(2 k+3)} R^{\frac{4 k+5-n}{n+1}}+\frac{M^{2}}{8 \pi R}
\end{aligned}
$$

where the second inequality in (82) follows from Lemma 3.3 and the bound $\mathcal{M}_{i}<M$ since $\mathcal{M}_{i} \in \Gamma^{M}$. Using (15) we observe that $\nu \equiv(4 k+5-n) /(n+1)>0$. We can set $R=\left\|\mathcal{M}_{1}-\mathcal{M}_{2}\right\|^{-1 / 2 \nu}$ in (82) to obtain

$$
\begin{aligned}
\left|\mathcal{E}\left(\mathcal{M}_{1}\right)-\mathcal{E}\left(\mathcal{M}_{2}\right)\right| & \leq \frac{(n+1)}{8 \pi(2 k+3)(4 k+5-n)}\left\|\mathcal{M}_{1}-\mathcal{M}_{2}\right\|^{\frac{1}{2}} \\
& +\frac{M^{2}}{8 \pi}\left\|\mathcal{M}_{1}-\mathcal{M}_{2}\right\|^{\frac{1}{2 v}}
\end{aligned}
$$

By Lemma 3.1 we obtain that there exists $C>0$ for which

$$
\left\|\mathcal{M}_{f}\right\| \leq C\|\| f\|\| ; \forall f \in \mathbf{B} .
$$

This and Lemma 3.5 imply that the potential energy $\mathcal{E}_{p}$, as defined in (27), is a continuous functional over $\mathbf{B}_{M}^{+}$. Moreover,

COROLLARY 3.6. - The energy-Casimir functional $\mathcal{D}$, defined in section 1.2, is continuous on $\mathbf{B}_{M}^{+}$for any $M>0$.

Let us define the functional $\mathcal{C}$ over $\Gamma^{M}$ :

$$
\mathcal{C}(\mathcal{M}) \equiv \mathcal{S}(\mathcal{M})+\mathcal{E}(\mathcal{M})
$$

where

$$
\begin{aligned}
\mathcal{S}(\mathcal{M}) & \equiv(4 \pi)^{-1 / n} \frac{n}{n+1}\|\mathcal{M}\|^{\frac{n+1}{n}} \\
& =(4 \pi)^{-1 / n} \frac{n}{n+1} \int_{0}^{\infty} r^{-2(k+1) / n}\left(\frac{d}{d r} \mathcal{M}\right)^{(n+1) / n} d r
\end{aligned}
$$


By Lemma 3.5 we obtain that $\mathcal{C}$ is continuous on $\Gamma^{M}$. Moreover, Lemma 3.1 and Lemma 3.2 yield

COROLlARY 3.7. - The inequality

$$
\mathcal{D}(f)-\mathcal{D}\left(f_{0}\right) \geq \mathcal{C}\left(\mathcal{M}_{f}\right)-\mathcal{C}\left(\mathcal{M}^{0}\right)
$$

holds for all $f \in \mathbf{B}_{0}^{+}$.

For any $R>0$, define

$$
\Gamma_{R}^{M} \equiv\left\{\mathcal{M} \in \Gamma^{M} ; \mathcal{M}(r)=M \quad \forall r>R\right\}
$$

Notice that, by the above definition, $\Gamma_{R}^{M} \subset \Gamma^{M}$ and, in particular, $\mathcal{M} \in \Gamma_{R}^{M}$ is defined for all $r>0$ (not only on the interval $[0, R]$ ). In addition, if $\mathcal{M} \in \Gamma_{R}^{M}$ then $\mathcal{M} \in \Gamma_{R^{\prime}}^{M}$ for any $R^{\prime}>R$.

Evidently

$$
\operatorname{cl}\left\{\cup_{R>0} \Gamma_{R}^{M}\right\}=\Gamma^{M}
$$

Moreover, if $f \in C_{b}^{s,+}(\mu, k)$, then $\mathcal{M}_{f} \in \Gamma_{R}^{M}$ for some $R>0$, related to the support radius of $f$. In particular:

$$
\mathcal{M}^{0} \in \Gamma_{R_{0}}^{M_{0}} \equiv \Gamma_{R_{0}}^{0} \subset \Gamma_{R}^{0} \text { for all } R \geq R_{0}
$$

where $R_{0}>0$ is the support radius of $\mathcal{M}^{0}$.

We now define the functional $\mathcal{C}_{R}$

$$
\mathcal{C}_{R}(\mathcal{M})=\mathcal{S}_{R}(\mathcal{M})+\mathcal{E}_{R}(\mathcal{M})
$$

where

$$
\begin{gathered}
\mathcal{S}_{R}(\mathcal{M}) \equiv(4 \pi)^{-1 / n} \frac{n}{n+1} \int_{0}^{R} r^{-2(k+1) / n}\left(\frac{d}{d r} \mathcal{M}\right)^{(n+1) / n} d r \\
\mathcal{E}_{R}(\mathcal{M})=-\frac{1}{8 \pi} \int_{0}^{R} \frac{\mathcal{M}^{2}(r)}{r^{2}} d r
\end{gathered}
$$

Comparing (87), (81) and (83) to (84) we obtain, for any $\mathcal{M} \in \Gamma_{R}^{M}$ :

$$
\mathcal{E}_{R}(\mathcal{M})=\mathcal{E}(\mathcal{M})+\frac{M^{2}}{8 \pi R} ; \mathcal{S}_{R}(\mathcal{M})=\mathcal{S}(\mathcal{M})
$$

The following is an immediate result of Corollary 3.7 and the above:

COROLlaRY 3.8. - For any $f \in C_{b, M_{0}}^{s,+}(\mu, k)$ there exists $\bar{R}>0$ so that

$$
\mathcal{D}(f)-\mathcal{D}\left(f_{0}\right) \geq \mathcal{C}_{R}\left(\mathcal{M}_{f}\right)-\mathcal{C}_{R}\left(\mathcal{M}^{0}\right)
$$

hold $\forall R>\bar{R}$. 


\section{THE EMDEN-FOWLER EQUATION REVISITED}

In this section, we discuss some properties of the Emden-Fowler equation and its E-solutions. We will obtain these solutions as minimizers of the functional $\mathcal{C}_{R}$ over $\Gamma_{R}^{0}$ for any $R \geq R_{0}$.

Recall that $\mathcal{M}^{0}$ is obtained in terms of an E-solution of the E-F equation via (12).

$$
\mathcal{M}^{0}(r)=4 \pi \int_{0}^{r} s^{2} \rho_{0}(s) d s=-4 \pi r^{2} \frac{d \phi_{0}}{d r}
$$

Recall also that $\mathcal{M} \in \Gamma_{R}^{0}$ for any $R \geq R_{0}$ (c.f. (85)), and $\phi_{0}\left(R_{0}\right)=0$.

The main result of this section is:

THeorem 4.1. - Let $R \geq R_{0}$ Then

$$
\mathcal{C}_{R}(\mathcal{M})-\mathcal{C}_{R}\left(\mathcal{M}^{0}\right) \geq 0
$$

for any $\mathcal{M} \in \Gamma_{R}^{0}$, and $\mathcal{C}_{R}(\mathcal{M})=\mathcal{C}_{R}\left(\mathcal{M}^{0}\right)$ only if $\mathcal{M}=\mathcal{M}^{0}$. Moreover, there exists $\delta^{\prime}=\delta^{\prime}(\varepsilon, R)>0$ whenever $\varepsilon>0$ and

$$
\mathcal{C}_{R}(\mathcal{M})-\mathcal{C}_{R}\left(\mathcal{M}^{0}\right) \geq \delta^{\prime}(\varepsilon, R)
$$

provided $\sup _{r \in[0, R]}\left|\mathcal{M}(r)-\mathcal{M}^{0}(r)\right| \geq c$.

The proof of Theorem 4.1 is obtained as a corollary of Lemma 4.2, 4.3 below and of Lemma 2.3 .

Let $0 \leq R_{1}<R$ and $0 \leq M_{1}<M_{0}$. Define

$$
\Gamma_{R, R_{1}}^{0, M_{1}}=\Gamma_{R}^{0} \cap\left\{\mathcal{M} ; \mathcal{M}\left(R_{1}\right)=M_{1}\right\}
$$

and

$$
\begin{gathered}
\mathcal{C}_{R, R_{1}}(\mathcal{M})=\mathcal{C}_{R}(\mathcal{M})-\mathcal{C}_{R_{1}}(\mathcal{M}) \\
=(4 \pi)^{-1 / n} \frac{n}{n+1} \int_{R_{1}}^{R} r^{-2(k+1) / n}\left(\frac{d}{d r} \mathcal{M}\right)^{(n+1) / r} d r \frac{1}{8 \pi} \int_{R_{\downarrow}}^{R} \frac{\mathcal{M}^{2}}{r^{2}}
\end{gathered}
$$

LEMMA 4.2. - The functional $\mathcal{C}_{R, R_{1}}$ is bounded from below on $\Gamma_{R, R_{1}}^{0, M_{1}}$. If $R_{1}>0$ or $R_{1}=M_{1}=0$ then a minimizer is attained.

Remark. - Notice that $\Gamma_{R, R_{1}}^{0, M_{1}}=\emptyset$ if $M_{1}>0$ and $R_{1}=0$. In this case, the first part of Lemma 4.2 is trivial since any functional is bounded on an empty set. If $R_{1}=M_{1}=0$, then $\Gamma_{R, R_{1}}^{0, M_{1}}=\Gamma_{R}^{0}$ and $\mathcal{C}_{R, R_{1}}=\mathcal{C}_{R}$. 
LEMMA 4.3. - Assume $R_{1}>0$ or $R_{1}=M_{1}=0$. Then any minimizer $\mathcal{M}$ of $\mathcal{C}_{R, R_{1}}$ over $\Gamma_{R, R_{1}}^{0, M_{1}}$ is given, in the interval $\left[R_{1}, R\right]$, by

$$
\mathcal{M}(r
$$

where $\phi$ is a solution of the E-F equation on the interval $\left[R_{1}, R\right]$ subjected to the boundary conditions:

$$
\text { (a) }\left.\frac{d \phi}{d r}\right|_{\left(R_{1}\right)}=-\frac{M_{1}}{4 \pi R_{1}^{2}} ; \quad \text { (b) }\left.\frac{d \phi}{d r}\right|_{(R)}=-\frac{M_{0}}{4 \pi R^{2}}
$$

If $R_{1}=M_{1}=0$ then $(92-a)$ is replaced by

$$
\lim _{r \rightarrow 0} r^{2} \frac{d \phi}{d r}=0
$$

In this section we only need Lemma 4.2 and Lemma 4.3 in the case $M_{1}=R_{1}=0$. We chose, however, to present these Lemmas in this general setting since it will be handy for the next section.

Before turning to the proofs, we introduce the Proposition below, which will be used in the proof of Lemma 4.2 as well as in section 5 .

Proposition 4.1. - Let $\mathcal{M} \in \Gamma_{R}^{M}$. For $\beta>0$ define

$$
\mathcal{M}_{\beta}(r)=\beta^{\gamma} \mathcal{M}(\beta r) ; \gamma \equiv \frac{2 k-n+3}{n-1}
$$

Then $\mathcal{M}_{\beta} \in \Gamma_{R / \beta}^{\beta^{\gamma} M}$ and

$$
\begin{aligned}
& \mathcal{S}_{R / \beta}\left(\mathcal{M}_{\beta}\right)=\beta^{2 \gamma+1} \mathcal{S}_{R}(\mathcal{M}) \\
& \mathcal{E}_{R / \beta}\left(\mathcal{M}_{\beta}\right)=\beta^{2 \gamma+1} \mathcal{E}_{R}(\mathcal{M}) .
\end{aligned}
$$

In particular

$$
\mathcal{C}_{R / \beta}\left(\mathcal{M}_{\beta}\right)=\beta^{2 \gamma+1} \mathcal{C}_{R}(\mathcal{M})
$$

Proposition 4.1 is verified by a direct substitution. Since the mapping (94) is one to one and onto from $\Gamma_{R}^{M}$ to $\Gamma_{R / \beta}^{\beta^{\gamma} M}$, we obtain immediately from Proposition 4.1

Corollary 4.2. - Suppose $\mathcal{M}$ is a minimizer of $\mathcal{C}_{R}$ over $\Gamma_{R}^{M}$. Then $\mathcal{M}_{\beta}$ is a minimizer of $\mathcal{C}_{R / \beta}$ over $\Gamma_{R / \beta}^{\beta^{\gamma} M}$. 
Proof of Lemma 4.2. - The case $R_{1}>0$ is almost trivial. We shall concentrate on the case $M_{1}=R_{1}=0$. By the remark below Lemma 4.2 we consider $\mathcal{C}_{R_{1}, R}=\mathcal{C}_{R}$ and $\Gamma_{R, R_{1}}^{0, M_{1}}=\Gamma_{R}^{0}$. We shall first prove that $\mathcal{C}_{R}$ is bounded from below and is coercive on $\Gamma_{R}^{M}$ for any $R>0, M>0$. That is

$$
\|\mathcal{M}\| \rightarrow \infty \Longrightarrow \mathcal{C}_{R}(\mathcal{M}) \rightarrow \infty
$$

We distinguish two cases:

a) $n<1$

b) $n>1$

Case (a) is the easier. Using Lemma 3.3 and (87)

$$
\begin{gathered}
-\mathcal{E}_{R}(\mathcal{M}) \leq\left(\frac{\|\mathcal{M}\|}{2 k+3}\right)^{2} \int_{0}^{R} r^{(4 k+6) /(n+1)-2} d r \\
=\frac{n+1}{4 k+5-n}\left(\frac{\|\mathcal{M}\|}{2 k+3}\right)^{2} R^{(4 k+5-n) /(n+1)} \equiv C(R)\|\mathcal{M}\|^{2}
\end{gathered}
$$

for any $\mathcal{M} \in \Gamma_{R}^{M}$. By (86),

$$
\begin{array}{r}
\mathcal{C}_{R}(\mathcal{M})=(4 \pi)^{-1 / n} \frac{n}{n+1}\|\mathcal{M}\|^{(n+1) / n}+\mathcal{E}_{R}(\mathcal{M}) \\
\geq(4 \pi)^{-1 / n} \frac{n}{n+1}\|\mathcal{M}\|^{(n+1) / n}-C(R)\|\mathcal{M}\|^{2}
\end{array}
$$

and (98) holds since $(n+1) / n>2$ by assumption.

Case (b) is more difficult since $(n+1) / n<2$ and the homogeneous inequality (100) is useless. However, for $\mathcal{M} \in \Gamma_{R}^{M}$ we have $\mathcal{M}(r) \leq$ $\mathcal{M}^{(n+1) / n} M^{1-1 / n}$, hence

$$
-\mathcal{E}_{R}(\mathcal{M}) \leq \frac{M^{(n-1) / n}}{8 \pi} \int_{0}^{R} r^{-2} \mathcal{M}^{(n+1) / n}(r) d r .
$$

By the Holder inequality

$$
\mathcal{M}(r)=\int_{0}^{r} \frac{d}{d t} \mathcal{M}(t) d t \leq\left(\int_{0}^{r}\left|\frac{d}{d t} \mathcal{M}(t)\right|^{(n+1) / n} d t\right)^{(n+1) / n} \cdot r^{1 /(n+1)}
$$

An application of (102) and an integration by parts on the r.h.s of (101) yields

$$
\begin{aligned}
& \quad-\mathcal{E}_{R}(\mathcal{M}) \\
& \leq \frac{M^{(n-1) / n}}{8 \pi} \int_{0}^{R} r^{(1-2 n) / n}\left(\int_{0}^{r}\left|\frac{d}{d t} \mathcal{M}(t)\right|^{(n+1) / n} d t\right) d r=\frac{n M^{(n-1) / n}}{8 \pi(n-1)} \\
& \\
& \quad\left(\int_{0}^{R}\left|\frac{d}{d t} \mathcal{M}(t)\right|^{(n+1) / n} t^{-(n-1) / n} d t-R^{(1-n) / n} \int_{0}^{R}\left|\frac{d}{d t} \mathcal{M}\right|^{(n+1) / n} d t\right) .
\end{aligned}
$$


Notice that $t^{-(n-1) / n} \leq t^{-2(k+1) / n}$ for $0<t \leq 1$ since $(n-1) / n<$ $2(k+1) / n$ (c.f. (15)). Thus

$$
\int_{0}^{R} t^{-(n-1) / n}\left|\frac{d}{d t} \mathcal{M}\right|^{(n+1) / n} \leq \int_{0}^{R} t^{-2(k+1) / n}\left|\frac{d}{d t} \mathcal{M}\right|^{(n+1) / n}
$$

provided $R \leq 1$. We obtain by (103)-(104) and (87) for $\mathcal{M} \in \Gamma_{R}^{M}, R \leq 1$,

$$
\begin{gathered}
\mathcal{S}_{R}(\mathcal{M}) \geq(4 \pi)^{-1 / n} \frac{n}{n+1} \int_{0}^{R} r^{-(n-1) / n}\left|\frac{d}{d t} \mathcal{M}(t)\right|^{(n+1) / n} d r \\
\geq(4 \pi)^{1-1 / n} \frac{n-1}{n+1} \frac{\mathcal{E}_{R}(\mathcal{M})}{M^{(n-1) / n}}
\end{gathered}
$$

Using the definition of $\mathcal{C}_{R}$

$$
\mathcal{C}_{R}(\mathcal{M})=\mathcal{S}_{R}(\mathcal{M})+\mathcal{E}_{R}(\mathcal{M}) \geq \mathcal{S}(\mathcal{M})\left[1-(4 \pi)^{1 / n-1} \frac{n+1}{n-1} M^{(n-1) / n}\right]
$$

Thus, there exists a positive constant $M^{c}$ so that $\mathcal{C}_{R}$ is nonnegative over $\Gamma_{R}^{M}$ provided $R \leq 1$ and $M \leq M^{c}$. Moreover, under the same conditions, there exists $C>0$ for which

$$
\mathcal{C}_{R}(\mathcal{M}) \geq C\|\mathcal{M}\|^{\frac{n+1}{n}}
$$

and (98) holds.

If $M \leq M^{c}$ but $R>1$, it follows by the definition of $\mathcal{C}_{R},(88)$ and (107)

$$
\begin{aligned}
& \mathcal{C}_{R}(\mathcal{M})= \mathcal{S}_{R}(\mathcal{M})+\mathcal{E}_{R}(\mathcal{M}) \geq \mathcal{S}_{1}(\mathcal{M})+\mathcal{E}_{1}(\mathcal{M})-\frac{M^{2}}{8 \pi} \\
& \quad+\left[\mathcal{S}_{R}(\mathcal{M})-\mathcal{S}_{1}(\mathcal{M})\right] \\
& \geq C \mathcal{S}_{1}(\mathcal{M})-\frac{M^{2}}{8 \pi}+\left[S_{R}(\mathcal{M})-\mathcal{S}_{1}(\mathcal{M})\right] \geq \min \{C, 1\} \mathcal{S}_{R}(\mathcal{M})-\frac{M^{2}}{8 \pi}
\end{aligned}
$$

To complete the proof of case (b), we apply Proposition 4.1 with $\beta$ chosen to satisfy $\beta^{\gamma} M=M^{c}$. Using (108) and (95) we obtain

$$
\mathcal{C}_{R}(\mathcal{M}) \geq \beta^{-(2 \gamma+1)}\left[\min \{C, 1\} \mathcal{S}_{R / \beta}\left(\mathcal{M}_{\beta}\right)-\frac{\left(M^{c}\right)^{2}}{8 \pi}\right]
$$

which reduce, after an application of (97) and substitution of $\beta^{\gamma}=M^{c} / M$, into

$$
\mathcal{C}_{R}(\mathcal{M}) \geq \min \{C, 1\}\|\mathcal{M}\|^{\frac{n+1}{n}}-C^{\prime}(M)
$$

for arbitrary $R>0$ and $M>0$.

Vol. $16, n^{\circ} \mathrm{I}-1999$ 
We conclude in particular that $\mathcal{C}_{R}$ is bounded from bclow on $\Gamma_{R}^{0}$ and is, moreover, coercive. To show the existence of a minimizer it is enough to show that $\mathcal{C}_{R}$ is weakly-lower-semi-continuous (w.l.s.c) with respect to the weak topology associated with the underlying space. The $\mathcal{S}_{R}$ part of $\mathcal{C}_{R}$ is a convex functional, hence it is w.l.s.c. We will show that $\mathcal{E}_{R}$ is weakly continuous .

Let $\mathcal{M}_{j} \rightarrow \mathcal{M}$ be a weakly converging sequence in $\Gamma_{R}^{0}$. By compact embedding of the associated weak topology in the $C^{0}$-norm it follows that $\mathcal{M}_{j}$ converge uniformly to $\mathcal{M}$ (remember that $\mathcal{M}(r)=M_{0}$ for all $r \geq R$ ). Define

$$
\mathcal{E}_{R}^{\varepsilon}(\mathcal{M}) \equiv-\int_{\varepsilon}^{R} \frac{\mathcal{M}^{2}(r)}{r^{2}} d r
$$

Then

$$
\lim _{j \rightarrow \infty} \mathcal{E}_{R}^{\varepsilon}\left(\mathcal{M}_{j}\right)=\mathcal{E}_{R}^{\varepsilon}(\mathcal{M})
$$

On the other hand, by Lemma 3.3

$$
\mathcal{E}_{R}^{\varepsilon}\left(\mathcal{M}_{j}\right)-\mathcal{E}_{R}\left(\mathcal{M}_{j}\right)=\int_{0}^{\varepsilon} \frac{\mathcal{M}_{j}^{2}(r)}{r^{2}} d r \leq C \varepsilon^{(4 k+5-n) /(n+1)}
$$

holds along the subsequence. Notice that $(4 k+5-n) /(n+1)>0$ by assumption (15), thus the r.h.s of (110) can be set arbitrarily small for a sufficiently small $\varepsilon>0$. Hence $\mathcal{E}_{R}\left(\mathcal{M}_{j}\right) \rightarrow \mathcal{E}_{R}(\mathcal{M})$ and $\mathcal{E}_{R}$ is weakly continuous on $\Gamma_{R}^{0}$.

Proof of Lemma 4.3. - We shall assume that $M_{1}=R_{1}=0$. The proof in the case $R_{1}>0$ is almost identical (and somewhat easier).

We will show that the E-F equation is the inverse of the Euler Lagrange equation (or inequality, in this case) corresponding to the functional $\mathcal{C}_{R}$.

Let $\mathcal{M}^{0, R} \in \Gamma_{R}^{0}$ be a minimizer of $\mathcal{C}_{R}$. Any $\mathcal{M} \in \Gamma_{R}^{0}$ can be identified with a continuous function. Moreover, its distributional derivative $\mathcal{M}^{\prime}$ is an $L_{(n+1) / n}\left(r^{-2(k+1) / n} d r\right)$ function, and $\mathcal{M}^{\prime} \geq 0$ a.e.

An admissible function $\omega \in L_{(n+1) / n}\left(r^{-2(k+1) / n} d r\right)$ is defined by the following condition: $\exists \delta>0$ so that

$$
\mathcal{M}_{\delta}^{\omega}(r) \equiv \mathcal{M}^{0}(r)+\delta \int_{0}^{r} \omega(s) d s \in \Gamma_{R}^{0}
$$

Given $\varepsilon>0, \varepsilon^{\prime}>0$, let

$$
\mathcal{A}_{\varepsilon^{\prime}}^{\varepsilon} \equiv\left\{r \in\left[\varepsilon^{\prime}, R\right],\left(\mathcal{M}^{0, R}\right)^{\prime} \leq \varepsilon \text { a.e. }\right\}
$$


It is easily verified that $\omega \in L_{\infty} \cap L_{(n+1) / n}\left(r^{-2(k+1) / n} d r\right)$ is admissible if

$$
\begin{gathered}
\omega(s)=0 \text { for } 0 \leq s \leq \varepsilon^{\prime}, \\
\int_{\varepsilon^{\prime}}^{R} \omega=0
\end{gathered}
$$

and

$$
\omega(s) \geq 0 \text { for almost any } s \in \mathcal{A}_{\varepsilon^{\prime}}^{\varepsilon} \text {. }
$$

Since $\mathcal{M}^{0, R}$ is a minimizer of $\mathcal{C}_{R}$ in $\bar{\Gamma}_{R}^{M}$, it follows that

$$
\begin{gathered}
0 \leq \mathcal{C}_{R}^{n, k}\left(\mathcal{M}^{\delta}\right)-\mathcal{C}_{R}^{n, k}\left(\mathcal{M}^{0, R}\right) \\
=\delta\left[(4 \pi)^{-1 / n} \int_{\varepsilon^{\prime}}^{R} r^{-2(k+1) / n}\left(\frac{d}{d r} \mathcal{M}^{0, R}\right)^{1 / n} \omega d r\right. \\
\left.-\frac{1}{4 \pi} \int_{\varepsilon^{\prime}}^{R} \int_{\xi^{\prime}}^{T} \omega(s) \frac{\mathcal{M}^{0, R}(r)}{r^{2}} d r d s\right]+o(\delta)
\end{gathered}
$$

We are permitted to apply integration by parts on the second integral of (115) and obtain

$$
\begin{gathered}
\frac{1}{4 \pi} \int_{\varepsilon^{\prime}}^{R} \int_{\varepsilon^{\prime}}^{r} \omega(s) \frac{\mathcal{M}^{0, R}(r)}{r^{2}} d s d r= \\
\int_{\varepsilon^{\prime}}^{R} U^{0, R}(r) \omega(r) d r-U^{0, R}\left(\varepsilon^{\prime}\right) \int_{0}^{\varepsilon^{\prime}} \omega(s) d s=\int_{\varepsilon^{\prime}}^{R} U^{0, R}(r) \omega(r) d r
\end{gathered}
$$

where we used (112) and the definition

$$
U^{0, R}(r) \equiv-\int_{r}^{R} \frac{\mathcal{M}^{0, R}(s)}{s^{2}} d s ; r \geq \varepsilon^{\prime}
$$

We obtain

$$
\int_{\varepsilon^{\prime}}^{R}\left[(4 \pi)^{-1 / n} r^{-2(k+1) / n}\left(\frac{d}{d r} \mathcal{M}^{0, R}(r)\right)^{1 / n}-U^{0, R}(r)\right] \omega(r) \geq 0
$$

for any permissible $\omega$ satisfying $(113,114)$. The function in the brackets of (118) can be identified as a function in $L_{n+1}\left(r^{-2(k+1) / n} d r\right)$, which is the dual of $L_{(n+1) / n}\left(r^{-2(k+1) / n} d r\right)$. It then follows by $(113,114)$ that

$$
(4 \pi)^{-1 / n} r^{-2(k+1) / n}\left(\frac{d}{d r} \mathcal{M}^{0, R}(r)\right)^{1 / n}-U^{0, R}(r) \geq \lambda
$$


must hold on $\left[\varepsilon^{\prime}, R\right]$, where $\lambda$ is some constant, while an equality in (119) is obtained at $\left[\varepsilon^{\prime}, R\right] \backslash \mathcal{A}_{\varepsilon^{\prime}}^{\varepsilon}$. Since $\varepsilon, \varepsilon^{\prime}$ can be chosen arbitrarily small, we obtain

$$
(4 \pi)^{-1} r^{-2} \frac{d}{d r} \mathcal{M}^{0, R}(r)=r^{2 k}\left(U^{0, R}(r)+\lambda\right)_{+}^{n}
$$

a.e. on $(0, R]$. In particular, $\mathcal{M}^{0, R}$ is continuously differentiable on $(0, R]$ and the equality in (120) holds anywhere. The proof now follows from (120) by the notation $U^{0, R}+\lambda \equiv \phi$ and the identity

$$
\text { (121) }-(4 \pi)^{-1} r^{-2} \frac{d}{d r} \mathcal{M}^{0, R}(r)=\left(\frac{d^{2}}{d r^{2}}+2 \frac{d}{d r}\right) U^{0, R}=\left(\frac{d^{2}}{d r^{2}}+2 \frac{d}{d r}\right) \phi
$$

obtained by (117).

Proof of Theorem 4.1. - We know by Lemma 4.2 that a minimizer $\mathcal{M}^{0, R}$ does exist for $\mathcal{C}_{R}$ on $\Gamma_{R}^{0}$. We also have the characterization of this minimizer by Lemma 4.3 , i.e

$$
\mathcal{M}^{0, R}=4 \pi r^{2} \phi^{\prime}
$$

where $\phi$ is a solution of the E-F equation. Since $\mathcal{M}^{0, R} \in \Gamma_{R}^{0}$, $\phi$ satisfy the boundary conditions (92 - b) and (93). Moreover, by Lemma 2.2, the condition (93) implies that $\phi$ is an E-solution on the interval $[0, R]$, namely

$$
\lim _{r \rightarrow 0} \phi(r) \equiv \phi(0)
$$

cxists. By Lemma 2.4 there exists at most one E-solution $\phi$ of the E-F equation compatible with $\left(92\right.$ b). Hence the minimizer $\mathcal{M}^{0, R}$ is unique. If $R>R_{0}$ then necessarily $\mathcal{M}^{0, R}=\mathcal{M}^{0}$.

To prove the second part of Theorem 4.1, let us consider a minimizing sequence $\mathcal{M}_{j} \in \Gamma_{R}^{0}$ of $\mathcal{C}_{R}$ subject to the constraint

$$
\sup _{r \in[0, R]}\left|\mathcal{M}_{j}(r)-\mathcal{M}^{0}(r)\right| \geq \varepsilon
$$

If (91) is violated, then $\lim _{j \rightarrow \infty} \mathcal{C}_{R}\left(\mathcal{M}_{j}\right)=\mathcal{C}_{R}\left(\mathcal{M}^{0}\right)$. The minimizing sequence $\left\{\mathcal{M}_{j}\right\}$ contains a weakly converging sequence. Let $\overline{\mathcal{M}}$ be the limit. Then $\overline{\mathcal{M}} \in \Gamma_{R}^{0}$. Since $\mathcal{C}_{R}$ is w.l.s.c, $\mathcal{C}_{R}\left(\mathcal{M}^{0, R}\right)=\mathcal{C}_{R}(\overline{\mathcal{M}})$. On the other hand the subsequence converge uniformly on $[0, R]$, thus

$$
\sup _{r \in[0, R]}\left|\overline{\mathcal{M}}(r)-\mathcal{M}^{0}(r)\right| \geq \varepsilon
$$

In particular $\overrightarrow{\mathcal{M}} \neq \mathcal{M}^{0}$. This violates the uniqueness of the minimizer in $\Gamma_{R}^{0}$. 


\section{PROOF OF THE MAIN RESULT}

\subsection{Uniform estimates}

In this subsection we will remove the $R$-dependence of $\delta^{\prime}$ in (91).

TIIEOREM 5.1. - For any $\varepsilon>0$ small enough, there exists a positive $\delta^{\prime}=\delta^{\prime}(\varepsilon)$ where

$$
\mathcal{C}_{R}(\mathcal{M})-\mathcal{C}_{R}\left(\mathcal{M}^{0}\right) \geq \delta^{\prime}(\varepsilon)
$$

holds for any $R>R_{0}$ and any $\mathcal{M} \in \Gamma_{R}^{0}$ provided $\sup _{r \geq 0}\left|\mathcal{M}(r)-\mathcal{M}^{0}(r)\right| \geq \varepsilon$.

Note that, unlike $\delta^{\prime}(\varepsilon, R)$ of Theorem $4.1, \delta^{\prime}$ of Theorem 5.1 is independent of $R$.

The proof of Theorem 5.1 follows from an application of Theorem 4.1 together with Lemma 5.2 and Lemma 5.3 below:

LEMMA 5.2. - For any $\varepsilon>0$ small enough, there exists a positive $\delta_{1}=\delta_{1}(\varepsilon)$ and $R_{1}=R_{1}(\varepsilon)>R_{0}$ for which

$$
\mathcal{C}_{R}(\mathcal{M})-\mathcal{C}_{R}\left(\mathcal{M}^{0}\right) \geq \delta_{1}(\varepsilon)
$$

for any $R>R_{1}(\varepsilon)$ and any $\mathcal{M} \in \Gamma_{R}^{0}$ which satisfy $\mathcal{M}\left(R_{1}\right) \leq M_{0}-\varepsilon$. Note that neither $\delta_{1}$ nor $R_{1}$ depends on $R$.

Lemma 5.3. - Let $\mathcal{M} \in \Gamma_{R}^{0}$ for some $R>R_{0}$. Assume $\sup _{r \geq 0}\left|\mathcal{M}(r)-\mathcal{M}^{0}(r)\right| \geq \varepsilon$ and $\mathcal{M}\left(R_{1}(\varepsilon)\right) \geq M_{0}-\varepsilon$ where $R_{1}(\varepsilon)$ as given in Lemma 5.2. Then there exists $\bar{R}=\bar{R}(\varepsilon) \geq R_{1}(\varepsilon)$ independent of $R$ and $\overline{\mathcal{M}} \in \Gamma_{\bar{R}}^{0}$ which satisfy

$$
\overline{\mathcal{M}}(r)=\mathcal{M}(r) \text { for } 0 \leq r \leq R_{1}(\varepsilon)
$$

and, moreover:

$$
\mathcal{C}_{R}(\overline{\mathcal{M}}) \leq \mathcal{C}_{R}(\mathcal{M})
$$

Proof of Theorem 5.1. - Let $\varepsilon>0$ and $\mathcal{M} \in \Gamma_{R}^{0}$ which satisfy the conditions of the Theorem. Then either of the alternatives hold:

(1) $\mathcal{M}\left(R_{1}(\varepsilon)\right) \leq M_{0}-\varepsilon$. Then, by Lemma 5.2, (122) holds with $\delta^{\prime}(\varepsilon)=\delta_{1}(\varepsilon)$, or

(2) $\mathcal{M}\left(R_{1}(\varepsilon)\right)>M_{0}-\varepsilon$. Then, by Lemma 5.3

$$
\mathcal{C}_{R}(\mathcal{M})-\mathcal{C}_{R}\left(\mathcal{M}^{0}\right) \geq \mathcal{C}_{R}(\overline{\mathcal{M}})-\mathcal{C}_{R}\left(\mathcal{M}^{0}\right)=\mathcal{C}_{\bar{R}}(\overline{\mathcal{M}})-\mathcal{C}_{\bar{R}}\left(\mathcal{M}^{0}\right)
$$

where the last inequality in (124) follows from $\overline{\mathcal{M}} \in \Gamma_{\bar{R}}^{0}$ since $\bar{R}>R_{0}$ by Lemma 5.3 . 
By the assumption of the second alternative, $\mathcal{M}(r)>M_{0}-\varepsilon=$ $\mathcal{M}^{0}(r)-\varepsilon$ for any $r>R_{1}(\varepsilon)$ (remember $R_{1}(\varepsilon)>R_{0}$ ). Since $\sup _{r \geq 0}\left|\mathcal{M}(r)-\mathcal{M}^{0}(r)\right| \geq \varepsilon$, there exists $0<r^{*} \leq R_{1}(\varepsilon) \leq \bar{R}(\varepsilon)$ for which $\left|\mathcal{M}\left(r^{*}\right)-\mathcal{M}^{0}\left(r^{*}\right)\right| \geq \varepsilon$. By Lemma 5.3, $\overline{\mathcal{M}} \in \Gamma_{\bar{R}}^{0}$ is identical with $\mathcal{M}$ on the interval $\left[0, R_{1}(\varepsilon)\right]$. Hence $\sup _{r \geq 0}\left|\overline{\mathcal{M}}(r)-\mathcal{M}^{0}(r)\right| \geq \varepsilon$. Then, Theorem 4.1 and (124) yield

$$
\mathcal{C}_{R}(\mathcal{M})-\mathcal{C}_{R}\left(\mathcal{M}^{0}\right) \geq \delta^{\prime}(\varepsilon, \bar{R}(\varepsilon))
$$

Combine both alternatives and set

$$
\delta^{\prime}(\varepsilon)=\min \left\{\delta_{1}(\varepsilon), \delta^{\prime}(\varepsilon, \bar{R}(\varepsilon))\right\} .
$$

This conclude the proof of the Theorem.

Proof of Lemma 5.2. - Let $\mathcal{M} \in \Gamma_{R}^{0}$ and $R_{1}>R_{0}$ which satisfy the assumption of the Lemma, namely $\mathcal{M}\left(R_{1}\right) \leq M_{0}-\varepsilon$. We shall find an explicit estimate for $R_{1}=R_{1}(\varepsilon)$ and $\delta_{1}(\varepsilon)$ for which (123) holds.

Since $\lim _{r \rightarrow \infty} \mathcal{M}(r)=M_{0}$, there must exist $R_{2} \geq R_{1}$ for which $\mathcal{M}\left(R_{2}\right)=M_{0}-\varepsilon$. We may, then, define $\mathcal{M}^{\varepsilon} \in \Gamma_{R_{2}}^{M_{0}-\varepsilon}$ by:

$$
\mathcal{M}^{\varepsilon}(r)=\mathcal{M}(r) \quad \forall r \in\left[0, R_{2}\right] ; \quad \mathcal{M}^{\varepsilon}=M_{0}-\varepsilon \text { for } r \geq R_{2}
$$

Evidently, $\mathcal{S}_{R}(\mathcal{M}) \geq \mathcal{S}_{R_{2}}\left(\mathcal{M}^{c}\right)$ and $\mathcal{E}_{R}(\mathcal{M}) \geq \mathcal{E}_{R_{2}}\left(\mathcal{M}^{c}\right)-M_{0}^{2} / 8 \pi R_{2}$. Hence

$$
\mathcal{C}_{R}(\mathcal{M}) \geq \mathcal{C}_{R_{2}}\left(\mathcal{M}^{\varepsilon}\right)-\frac{M_{0}^{2}}{8 \pi R_{2}}
$$

In addition, $\mathcal{C}_{R}\left(\mathcal{M}^{0}\right) \leq \mathcal{C}_{R_{2}}\left(\mathcal{M}^{0}\right)+M_{0}^{2} / 8 \pi R_{2}$ (notice that $\mathcal{M}^{0} \in \Gamma_{R \text {, }}^{0}$ since $R_{2} \geq R_{1} \geq R_{0}$ by assumption). Thus

$$
\mathcal{C}_{R}(\mathcal{M})-\mathcal{C}_{R}\left(\mathcal{M}^{0}\right) \geq \mathcal{C}_{R_{2}}\left(\mathcal{M}^{\varepsilon}\right)-\mathcal{C}_{R_{2}}\left(\mathcal{M}^{0}\right)-\frac{M_{0}^{2}}{4 \pi R_{2}}
$$

Let us consider now the minimizer of $\mathcal{C}_{R_{2}}$ over $\Gamma_{R_{2}}^{M_{0}-\varepsilon}$. By Theorem 4.1 we know that this minimizer exists. We may, now, call Corollary 4.2 and observe that $\mathcal{M}^{\varepsilon}=\mathcal{M}_{\beta}^{0}$ provided $\beta R_{2}>R_{0}$ and

$$
\mathcal{M}_{\beta}^{0}\left(R_{2}\right)=\beta^{\gamma} M_{0}=M_{0}-\varepsilon .
$$

Let

$$
\beta=\left(1-\frac{\varepsilon}{M_{0}}\right)^{1 / \gamma}
$$


If $\varepsilon$ is small enough, then $\beta R_{2}>R_{0}$, and (128) implies (127). We now recall Proposition 4.1 , (97) to obtain

$$
\mathcal{C}_{R_{2}}\left(\mathcal{M}_{\beta}^{0}\right)=\beta^{2 \gamma+1} \mathcal{C}_{\beta R_{2}}\left(\mathcal{M}^{0}\right) \geq \beta^{2 \gamma+1} \mathcal{C}_{R_{2}}\left(\mathcal{M}^{0}\right)-\frac{M_{0}^{2}}{4 \pi R_{2}}
$$

Since $\mathcal{M}_{\beta}^{0}$ is the minimizer of $\mathcal{C}_{R_{2}}$ over $\Gamma_{R_{2}}^{M_{0}-\varepsilon}$ and $\mathcal{M}^{\varepsilon} \in \Gamma_{R_{2}}^{M_{0}-\varepsilon}$

$$
\mathcal{C}_{R_{2}}\left(\mathcal{M}^{\varepsilon}\right) \geq \mathcal{C}_{R_{2}}\left(\mathcal{M}_{\beta}^{0}\right)
$$

Combining (126), (129) and (130)

$$
\mathcal{C}_{R}(\mathcal{M})-\mathcal{C}_{R}\left(\mathcal{M}^{0}\right) \geq\left(\beta^{2 \gamma+1}-1\right) \mathcal{C}_{R_{2}}\left(\mathcal{M}^{0}\right)-\frac{M_{0}^{2}}{2 \pi R_{2}}
$$

Substitute (128) in (131) to obtain

$$
\mathcal{C}_{R}(\mathcal{M})-\mathcal{C}_{R}\left(\mathcal{M}^{0}\right) \geq-\mathcal{C}_{R_{2}}\left(\mathcal{M}^{0}\right)\left[1-\left(1-\frac{\varepsilon}{M_{0}}\right)^{\nu}\right]-\frac{M_{0}^{2}}{2 \pi R_{2}}
$$

where

$$
\nu \equiv \frac{4 k+5-n}{2 k+3-n}>0
$$

(c.f. (15)). To complete the proof of the Lemma, we show that

$$
\lim _{R \rightarrow \infty} \mathcal{C}_{R}\left(\mathcal{M}^{0}\right)<0
$$

Granted (133) and recalling that $\mathcal{C}_{R}\left(\mathcal{M}^{0}\right)$ is monotone decreasing as a function of $R$ for $R>R_{0}$, we may choose $R_{1}=R_{1}(\varepsilon)$ sufficiently large for which

$$
\begin{gathered}
-\mathcal{C}_{R_{2}}\left(\mathcal{M}^{0}\right)\left[1-\left(1-\frac{\varepsilon}{M_{0}}\right)^{\nu}\right]-\frac{M_{0}^{2}}{2 \pi R_{2}} \\
\geq-\mathcal{C}_{R_{1}}\left(\mathcal{M}^{0}\right)\left[1-\left(1-\frac{\varepsilon}{M_{0}}\right)^{\nu}\right]-\frac{M_{0}^{2}}{2 \pi R_{1}} \equiv \delta_{1}(\varepsilon)>0
\end{gathered}
$$

for all $R_{2}>R_{1}(\varepsilon)$. The inequality (122) follows from (132), (134).

We turn now to the proof of (133). Suppose $\mathcal{C}_{R}\left(\mathcal{M}^{0}\right) \geq 0$ for all $R$ sufficiently large. Then $\mathcal{M}_{\beta}^{0}$ is a minimizer of $\mathcal{C}_{R / \beta}$ over $\Gamma_{R / \beta}^{\beta^{\gamma}} M_{0} \quad \forall \beta>0$. Using Proposition 4.1 (97)

$$
\mathcal{C}_{R / \beta}\left(\mathcal{M}_{\beta}^{0}\right)=\beta^{2 \gamma+1} \mathcal{C}_{R}\left(\mathcal{M}^{0}\right) \geq 0
$$

Vol. $16, n^{\circ} 1-1999$ 
for any $\beta>0$. On the other hand, $\beta^{\gamma} \mathcal{M}^{0} \in \Gamma_{R}^{\beta^{\gamma} M_{0}}$ for any $R \geq R_{0}$. By the definition of $\mathcal{C}_{R}$

$$
\mathcal{C}_{R}\left(\beta^{\gamma} \mathcal{M}^{0}\right)=\beta^{\gamma(1+1 / n)} \mathcal{S}_{R}\left(\mathcal{M}^{0}\right)+\beta^{2 \gamma} \mathcal{E}_{R}\left(\mathcal{M}^{0}\right)
$$

where $\mathcal{S}_{R}\left(\mathcal{M}^{0}\right)>0$ and $\mathcal{E}_{R}\left(\mathcal{M}^{0}\right)<0$ for any $R>0$ as well as in the limit $R \rightarrow \infty$ by definition $(86,87$ ). In the case $n>1$ (where $\gamma>0$ ), (136) guarantees that $\inf _{\Gamma_{R}^{M}} \mathcal{C}_{R}<0$ where $M=\beta^{\gamma} M_{0}$ and $\beta$ sufficiently large (equivalently $M$ sufficiently large). This contradicts (135) via the minimality of $\mathcal{C}_{R / \beta}\left(\mathcal{M}_{\beta}^{0}\right)$ over $\Gamma_{R}^{\beta^{\gamma} M_{0}}$ for $R>R_{0} / \beta$. In the case $n<1(\gamma<0)$ we obtain a contradiction by taking large $\beta$ (corresponding, this time, to small $M$ ) in (136), which makes the second term in (136) dominant.

Proof of Lemma 5.3 - Consider the functional $\mathcal{C}_{R}$ defined over the set

$$
\Lambda \equiv\left\{\mathcal{N} \in \mathcal{M} \in \Gamma_{R}^{0} ; \mathcal{N}(r)=\mathcal{M}(r) \text { for } 0 \leq r \leq R_{1}(\varepsilon)\right\}
$$

By Lemma 4.2 and Lemma 4.3 it follows that there exists a minimizer $\overline{\mathcal{M}} \in \mathcal{N}$ for $\mathcal{C}_{R}$ which satisfy

$$
\begin{aligned}
& \overline{\mathcal{M}}(r)=-4 \pi r^{2} \frac{d \phi_{R}}{d r} \\
& \text { for } r \in\left[R_{1}(\varepsilon), R\right] ; \overline{\mathcal{M}}(r)=\mathcal{M}(r) \text { for } r \in\left(0, R_{1}(\varepsilon)\right]
\end{aligned}
$$

where $\phi_{R}$ is a solution of the E-F equation on the interval $\left[R_{1}(\varepsilon), R\right]$ which satisfy the boundary conditions:

$$
-\left.4 \pi R_{1}^{2} \frac{d \phi_{R}}{d r}\right|_{\left(r=R_{1}\right)} \in\left[M_{0}-\varepsilon, M_{0}\right] ;-\left.4 \pi R^{2} \frac{d \phi_{R}}{d r}\right|_{(r=R)}=M_{0}
$$

The Lemma will follows provided we show that there exists $\bar{R}>R_{1}$ for which all solutions of the E-F equation subjected to (137) where $R>\bar{R}$ do satisfy $\phi_{R}(\bar{R})<0$. Assuming the opposite, there exists a sequence $R_{j} \rightarrow \infty$ and $\bar{R}_{j} \rightarrow \infty$ where $\bar{R}_{j} \leq R_{j}$ and $\phi_{R_{j}} \geq 0$ on the intervals $\left[R_{1}, R_{j}\right]$. Let us fix an $R_{*}>R_{1}$. By (137) it follows that $-4 \pi R_{*}^{2} \phi_{R_{j}}^{\prime}\left(R_{*}\right) \in\left[M_{0}-\varepsilon, M_{0}\right]$ provided $R_{j}>R_{*}$. By Lemma 2.4 we obtain that $\phi_{R_{j}}\left(R_{1}\right)$ are uniformly bounded. Thus, we may extract a subsequence for which $\phi_{R_{j}}\left(R_{1}\right)$ converge to, say, $\alpha_{\infty}$, while $-4 \pi R_{1}^{2} \phi_{R_{j}}^{\prime}\left(R_{1}\right)$ converge to $\beta \in\left[M_{0}-\varepsilon, M_{0}\right]$. Let $\phi_{\infty}$ be the solution of the E-F equation on the interval $\left[R_{1}, \infty\right)$ subjected to

$$
\phi_{\infty}\left(R_{1}\right)=\alpha_{\infty} \quad ; \quad-\left.4 \pi R_{1}^{2} \frac{d \phi_{\infty}}{d r}\right|_{\left(r=R_{1}\right)}=\beta
$$


It follows that the subsequence $\phi_{R_{j}}$ converge locally uniformly to $\phi_{\infty}$. By the assumption $\phi_{R_{j}} \geq 0$ on the intervals $\left[R_{1}, R_{j}\right]$ we obtain that

$$
\phi_{\infty} \geq 0 ; \forall r \geq R_{1} \text {. }
$$

However, by Lemma 2.1, all solutions $\phi$ of the E-F equation have a finite radius. This implies that there exists $\bar{R}>R_{1}$ for which $\phi_{\infty}(\bar{R})=0$. Evidently, $\phi_{\infty} \not \equiv 0$ hence $\phi_{\infty}(r)<0$ for any $r>\bar{R}$. This contradicts (138).

\subsection{Proof of the stability Theorem}

We are now in a position to prove the main result Theorem 1.1. First, we show that Theorem 1.1 is implied by Theorem 1.2.

Let $\delta>0$ be sufficiently small for (20) to imply that $\left|\mathcal{D}(\hat{f})-\mathcal{D}\left(f_{0}\right)\right|<$ $\delta^{\prime}(\varepsilon)$, where $\delta^{\prime}$ as given in Theorem 1.2-(5). This is possible since $\mathcal{D}$ is continuous by Theorem 1.2-(3). Now, if (21) is violated for some $t>0$, then we obtain a contradiction to (25) since $\mathcal{D}$ is time invariant.

The proof of Theorem 1.2-(1) and the time invariance of the energyCasimir was discussed in section 1.2. Evidently, $f(\cdot, t) \in \mathbf{B}_{0}^{+}$since $f(\cdot, t) \in C_{b}^{s,+}(\mu, k)$ by the classical existence Theorem [3]. Part (2) is proved by Lemma 3.2. The continuity of $\mathcal{D}$ [part (3)] was proved in Corollary 3.6. Part (4) follows from Lemma 3.1. As for part (5), it follows from Theorem 5.1 and Corollary 3.8, since $C_{M_{0}, b}^{s,+}$ is dense in $\mathbf{B}_{0}^{+}$.

\section{ACKNOWLEDGMENTS}

I wish to express special thanks to Prof. J. Batt and Dr. G. Rein from the University of Munich for their deep interest in result presented, for the helpful discussion we had while I was visiting Munich on July 96 and for their warm and encouraging attitude. The current research is a part of a project supported by the U.S-Israel Bi-National Foundation.

\section{REFERENCES}

[1] A. V. Antonov, Remarks on the problems of stability in stellar dynamics, Soviet Astr, AJ., Vol. 4, 1961, pp. 859-867.

[2] A. V. ANTONOV, Solution of the problem of stability of a stellar system with the Emden density law and spherical velocity distribution, J. Leningrad Univ. Ser. Mekh. Astro., 1962, Vol. 7, pp. 135-146. 
[3] J. BAT,, Global symmetric solutions of the initial value problem of stellar dynamics, J. Diff Eq., 1977, Vol. 25, pp. 342-364.

[4] J. BAT, W. FALTENBaCHER and E. HORST, Stationary spherically symmetric models in stellar dynamics, Arch. for Mech. and Anal. , 1986, Vol. 93, pp. 159-183.

[5] J. BATT and K. Pfaffelmoser, On the radius continuity of the models of Polytropic gas spheres which corresponds to the positive solutions of the generalized Emden-Fowler equation, Math. Meth. in the Appl. Sci., 1988, Vol. 10, pp. 499-5l6.

[6] J. BATT and G. REIN, A rigorous stability result for the Vlasov-Poisson system in three dimensions, Anal. di Mat. Pura ed Applicata, 1993, Vol. CLXIV, pp. 133-154.

[7] J. Binney and S. Tremaine. Galactic Dynamics, Princeton University Press, Princeton, 1987.

[8] A. M. Fridman and V. L. PolyaChenko, Physics of Gravitating Systems, Vol. I\&II, Springer Verlag, New York, Berlin, Heidelberg, Tokyo, 1984.

[9] D. D. Holm, J. E. Maksuen, T. Ratiu and A. Weinstein, Nonlinear stability of fluid and plasma equilibria, Physics Reports, 1985, Vol. 123, Nos. 1 and 2, pp. 1-116.

[10] C. MARChIoro and M. Pulvirentr, A note on the nonlinear stability of a spatially symmetric Vlasov-Poisson flow Math. Meth. in the Appl. Sci., 1986, Vol. 8, pp. 284-288.

[11] G. ReIN, Nonlinear stability for the Vlasov Poisson system; the energy-Casimir method, preprint.

[121 J. SchaEfFER, Global existence of smooth solutions to the Vlasov-Poisson system in three dimensions, Commun. Part. Diff. Eqns., 1991, Vol. 16, pp. 1313-1335.

[13] W. J. Van den Broek and F. Verhulst, A generalized Lane-Emden-Fowler equation, Math. Meth. in the Appl. Sci., 1982, Vol. 4, pp. 259-271.

[14] Y. H. WAN, Nonlinear stability of stationary spherically symmetric models in stellar dynamics, Arch. Rational. Mech. Anal., 1990, Vol. 112, pp. 83-95.

[15] J. S. WonG, On the generalized Emden-Fowler equation, S.I.A.M. Review, 1975, Vol. 17, pp. 339-360.

(Manuscript received Octoher 30, 1996.) 